\title{
Robust Unsupervised Factory Activity Recognition with Body-worn Accelerometer Using Temporal Structure of Multiple Sensor Data Motifs
}

\author{
QINGXIN XIA, Osaka University, Japan \\ JOSEPH KORPELA, Osaka University, Japan \\ YASUO NAMIOKA, Toshiba Corporation, Japan \\ TAKUYA MAEKAWA*, Osaka University, Japan
}

\begin{abstract}
This paper presents a robust unsupervised method for recognizing factory work using sensor data from body-worn acceleration sensors. In line-production systems, each factory worker repetitively performs a predefined work process with each process consisting of a sequence of operations. Because of the difficulty in collecting labeled sensor data from each factory worker, unsupervised factory activity recognition has been attracting attention in the ubicomp community. However, prior unsupervised factory activity recognition methods can be adversely affected by any outlier activities performed by the workers. In this study, we propose a robust factory activity recognition method that tracks frequent sensor data motifs, which can correspond to particular actions performed by the workers, that appear in each iteration of the work processes. Specifically, this study proposes tracking two types of motifs: period motifs and action motifs, during the unsupervised recognition process. A period motif is a unique data segment that occurs only once in each work period (one iteration of an overall work process). An action motif is a data segment that occurs several times in each work period, corresponding to an action that is performed several times in each period. Tracking multiple period motifs enables us to roughly capture the temporal structure and duration of the work period even when outlier activities occur. Action motifs, which are spread throughout the work period, permit us to precisely detect the start time of each operation. We evaluated the proposed method using sensor data collected from workers in actual factories and achieved state-of-the-art performance.
\end{abstract}

CCS Concepts: • Human-centered computing $\rightarrow$ Ubiquitous and mobile computing theory, concepts and paradigms; - Mathematics of computing $\rightarrow$ Sequential Monte Carlo methods; Time series analysis;

Additional Key Words and Phrases: Activity recognition, wearable sensor, factory work

ACM Reference Format:

Qingxin Xia, Joseph Korpela, Yasuo Namioka, and Takuya Maekawa. 2020. Robust Unsupervised Factory Activity Recognition with Body-worn Accelerometer Using Temporal Structure of Multiple Sensor Data Motifs. Proc. ACM Interact. Mob. Wearable Ubiquitous Technol. 4, 3, Article 97 (September 2020), 30 pages. https://doi.org/10.1145/3411836

"This is the corresponding author

This work is partially supported by JST CREST JPMJCR15E2, JSPS KAKENHI Grant Number JP16H06539 and JP17H04679. The first author is supported by China Scholarship Council.

Authors' addresses: Qingxin Xia, Osaka University, Graduate School of Information Science and Technology, Suita, Osaka, 5650871, Japan; Joseph Korpela, Osaka University, Graduate School of Information Science and Technology, Suita, Osaka, 5650871, Japan; Yasuo Namioka, Toshiba Corporation, Corporate Manufacturing Engineering Center, Yokohama, Kanagawa, 2350017, Japan; Takuya Maekawa, takuya.maekawa@acm.org,maekawa@ist.osaka-u.ac.jp, Osaka University, Graduate School of Information Science and Technology, Suita, Osaka, 5650871, Japan.

ACM acknowledges that this contribution was authored or co-authored by an employee, contractor, or affiliate of the United States government. As such, the United States government retains a nonexclusive, royalty-free right to publish or reproduce this article, or to allow others to do so, for government purposes only.

(c) 2020 Association for Computing Machinery.

2474-9567/2020/9-ART97 \$15.00

https://doi.org/10.1145/3411836

Proc. ACM Interact. Mob. Wearable Ubiquitous Technol., Vol. 4, No. 3, Article 97. Publication date: September 2020. 


\section{INTRODUCTION}

\subsection{Background}

Human activity recognition using sensor data obtained from body-worn smart devices such as smartphones, smartwatches, and smart glasses has been actively studied in the ubicomp and wearable-computing research communities [12, 14, 23, 24, 26,31,36], because activity recognition using these sensors can be applied to a variety of applications in both home settings, e.g., analyzing the daily life of elderly patients, and industrial settings, e.g., quality assurance for factory workers. This study focuses on the use of activity recognition in industrial settings, namely on the recognition of work done in factories by workers that are wearing acceleration sensors. One common feature found in such industrial settings is the line production system, in which each product passes through the same sequence of work processes in series with workers positioned at various stages along the line repetitively performing predefined sequences of operations, such as removing film from a board and then screwing parts onto the board. Because such manual processes still constitute the core of line production systems, the improvement of manual labor is one of the most important aspects of increasing productivity in these systems $[2,19]$.

Generally speaking, activity recognition refers to the task of estimating the activity class performed by a target person during a given period of time. In the case of factory activity recognition, the activity classes correspond to each of operations in a work process and the time periods can be chosen so that we can detect the start and end times for each operation. Having detected the timing of the operations, we can then support many useful applications, such as monitoring the status of workers and detecting outlier activities. Because large numbers of workers are often involved in line production systems, it can be difficult for line managers to monitor all of their workers simultaneously; therefore, automated monitoring systems for factory-work activities are in high demand by manufacturers, with several previous studies in the ubicomp community having proposed solutions that are based on supervised activity recognition $[1,3-5,13]$. However, collecting labeled training data for supervised learning can be prohibitively costly when applied to factory-work activities due to the following reasons:

(1) Training data must be collected for every worker because each worker often performs a different sequence of operations.

(2) Modern production systems are revised frequently (e.g., weekly or monthly) to address the ever-changing needs of customers. Therefore, the operations themselves are also modified frequently, necessitating the collection of training data on a regular basis.

\subsection{Unsupervised Factory Activity Recognition}

To address the issues encountered when using supervised learning approaches, unsupervised factory activity recognition techniques have also been studied in the ubicomp community. For example, Maekawa et al. [25] measured the duration of each work period, i.e., the entire sequence of operations through which a product passes on a production line, in an unsupervised manner. Xia et al. [38] took this a step further by recognizing the individual operations conducted within the work periods along with their starting and ending times with the help of process instructions, which are documents that describe the flow of operations included in each work period along with their standard time durations. However, these previous unsupervised approaches are not robust to the outlier activities that usually occur in real-world factory work. These outlier activities occur when factory workers stop their work for reasons such as: (i) walking to another position to pick something up (e.g., tools or materials), to socialize with another worker, or to use washing facilities, (ii) waiting for a product to arrive at their station, possibly due to a delay caused by a previous worker in the line, and (iii) responding to inquiries from either another worker or a manager that visits their station. 


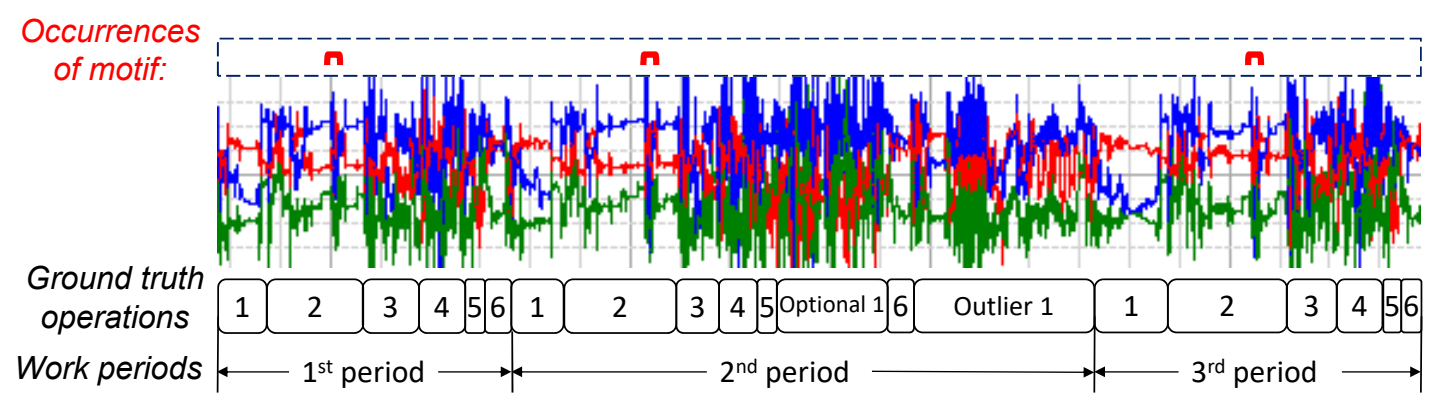

Fig. 1. Example acceleration data collected from a worker. The red, green, and blue lines show the $x^{-}, y^{-}$, and $z$-axis data, respectively. The rectangles below show the ground truth labels of the operations. The duration of each operation period is about $59 \mathrm{~s}$. Each work period consists of six operations, which are defined in the process instructions. The red brackets show the occurrences of an example motif. "Optional 1" shows an optional operation.

The unsupervised approaches mentioned above do not perform well when outlier activities are included in the sensor data, because they rely on being given information about any included activities in advance. That is, those previous methods relied on a single sensor data motif ${ }^{1}$ that appeared only once per work period and tracked the occurrences of that motif using a particle filter. They estimated the next occurrence of the motif based on a predefined standard lead time (i.e., the standard duration of one work period), meaning that those methods could not track the motif when long outlier activities were included in the sensor data.

Figure 1 shows an example of acceleration data collected from a factory worker's smartwatch. In this example, a typical series of operations are iterated three times, with each iteration (i.e., work period) comprising a sequence of six operations. This figure also shows the occurrences of an example sensor data motif (indicated by the red brackets). In this example, the motif corresponds to an action characteristic of the second operation, which is performed only once per work period. Here we can see that because outlier activities occur after the sixth operation in the second work period, the intervals between occurrences of the motif become irregular, making it difficult to estimate the third occurrence of the motif using only our prior information (i.e., standard lead time). In addition, it is impossible to know where in the sequence of operations that the outlier activities occurred, making it difficult to estimate the start times for the individual operations. Therefore, methods that rely on tracking a single motif based on a predefined standard lead times can break down when encountering such outlier activities.

\subsection{Approach}

In this study, we have developed a robust unsupervised factory activity recognition method that leverages temporal relationships between multiple motifs that appear in each work period. Specifically, we differentiate between two kinds of motifs in the proposed method: period motifs and action motifs, and leverage multiple period motifs and action motifs to achieve robust recognition. In this study, the term period motif refers to a unique data segment that occurs only once in each work period, e.g., data corresponding to a hammering action that is performed only once in each work period. The term action motif refers to a data segment that occurs several times in each work period, e.g., data corresponding to a common repetitive action such as screwing.

In the previous methods, only a single period motif was tracked, with each subsequent location of the motif estimated as being around one standard lead time after the previous occurrence of the motif and any candidate data segments at that estimated location verified based on the similarity between the sensor data of the candidate

\footnotetext{
${ }^{1}$ In this case, a sensor data motif is a unique data segment that corresponds to a particular action conducted during one of the work processes.
} 


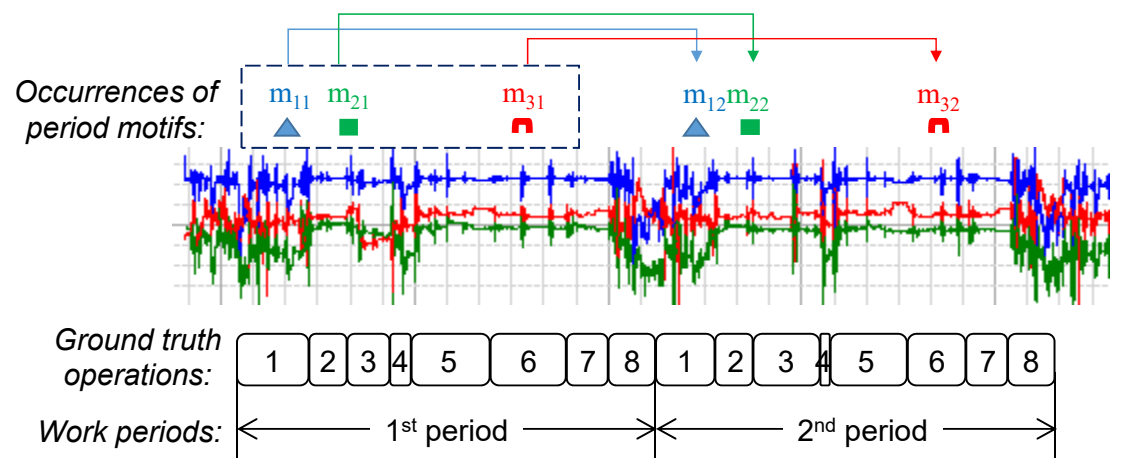

Fig. 2. Example acceleration data collected from a worker. The duration of each operation period is about $130 \mathrm{~s}$. Each work period consists of eight operations, which are defined in the process instructions. Three period motifs are extracted in this example. $m_{x y}$ shows the $y$-th occurrence of the $x$-th motif.

and the sensor data of the original occurrence of the motif. However, this approach, which relies on the standard lead time, is not robust in the presence of outlier activities. In contrast, our method concurrently tracks multiple period motifs, detecting the candidate locations for each motif by monitoring the sensor data using a computationally efficient method, and then verifies each possible occurrence of a motif by using not only the standard lead time of a work period, but also the temporal relationship between that occurrence of the current period motif and the neighboring occurrences of other period motifs. Finally, we incorporate the temporal structure of multiple period motifs into a particle filter in order to achieve a robust motif tracking algorithm.

By leveraging multiple period motifs per work period, our method is able to capture the rough structure of each work period in a robust way. For example, assume that we have a work sequence that consists of eight operations, where we have extracted period motifs from the first, second and sixth operations as shown in Figure 2. If an outlier activity were to occur between the first and second operations, we would roughly know that the outlier segment exists between the first and second operations based on the temporal relationship (temporal distance) between the period motifs of the first and second operations, enabling us to roughly estimate the ending time of the first operation and starting time of the second operation. In this way, leveraging the temporal structure of multiple motifs enables us to improve the robustness of the recognition process. The temporal structure of the period motifs can also be used to robustly track multiple period motifs. In the example shown in Figure 2, assume that it is difficult to detect the occurrence of motif $m_{22}$ based on the sensor data due to noise in the sensor data. In this case, we can roughly locate motif $m_{22}$ by using the temporal relationships that were observed in previous work periods between $m_{2}$ and $m_{1}$ and between $m_{2}$ and $m_{3}$.

Along with the period motifs described above, our method also incorporates information derived from action motifs. Action motifs occur much more frequently within any given work period than period motifs, because action motifs correspond to repetitive actions such as screwing. Therefore, good action motifs can be used to understand the fine-scale structure of a work period (i.e., the start time of each operation). When estimating the start time for each operation within a work period, we can then combine information about the locations of occurrences of action motifs with information about the locations of period motifs, information about trend changes in the sensor data, and information from the process instructions. Specifically, we prepare a secondary particle filter that is responsible for tracking the start time of each operation by leveraging the above information as follows. 
- The transition points between operations in a work period may contain noticeable trend changes in the sensor data. For example, in Figure 2, we can see a dramatic change in the characteristics of the sensor data at the end of the first operation in each work period. Therefore, we employ a nonparametric Bayesian hidden Markov model (HMM) to detect trend changes, which indicate potential transition points in the activities. We then determine the best sequence of transition points (i.e., operation start times) based on the trend changes by matching them to the standard operation durations described in the process instructions.

- Because several period and action motifs are observed in each work period, the temporal relationships between the start time of an operation and the occurrences of period and action motifs can be used to estimate the start time of the operation. For example, in Figure 2, the start times of the third operation can be estimated as being just after occurrences of the second period motif (i.e., $m_{21}$ and $m_{22}$ ). However, in the case of action motifs, because they can occur many times within each work period, it is difficult to precisely track each of its occurrences. Therefore, we instead feed simple statistical features related to occurrences of each action motif (e.g., the number of estimated occurrences between the estimated start and end times of an operation) into the secondary particle filter.

\subsection{Contributions}

The research contributions of this study are summarized as follows.

- We propose a robust unsupervised factory activity recognition method that makes use of the temporal structure of two types of motifs: period motifs and action motifs.

- Our method showed significant improvement in activity recognition accuracy over previous unsupervised methods when using real-world sensor data collected from factory workers, even when outliers were included in the sensor data.

- As a side effect of our method for tracking multiple motifs, we can estimate the start times of operations with lower computation costs than the previous method that relied on the dynamic time warping (DTW) [32], with our method showing a significant improvement in computation speed when using real-life sensor data collected in a factory.

The proposed method should outperform the previous factory activity recognition method [38], which employed a single period motif, due to the following novel features of the proposed method. (i) Because the proposed method tracks multiple period motifs, we can estimate the start times of operations more precisely using the temporal relationship between the occurrences of period motifs, which are densely observed, and the operation start times. In addition, the temporal distances between the multiple period motifs can be used to detect the occurrences of outlying activities. (ii) Statistical features extracted based on the occurrences of action motifs (e.g., information corresponding to the number of occurrences of an action motif) permit us to calculate the similarity between sensor data segments, which is used to compare data segments with those from previous periods, with smaller computation costs than the previous method, which relied on DTW. By using our novel idea of leveraging two types of motifs during unsupervised factory activity recognition as listed above, we were able to improve recognition accuracy by $36 \%$ for work processes containing outliers and reducing computing costs by $86 \%$.

In the rest of this paper, we first introduce work related to activity recognition including factory activity recognition. Then, we describe the design of our method for factory work recognition. Finally, in the evaluation section, we test our method using real-world sensor data collected in factories.

\section{RELATED WORK}

Due to the recent advances in sensing technologies, smartwatches and wristbands equipped with various sensors such as acceleration sensors, microphones, cameras, and heart rate sensors are now commercially available, 
with these types of wearable sensors being used in various studies on recognizing human activities. In particular, human activity recognition using acceleration sensors is a core topic in the ubicomp community because accelerometers have a good trade-off between prediction accuracy and minimal device-maintenance overhead. Many prior examples have applied supervised learning techniques on acceleration data in order to recognize daily activities $[8,17,21,22]$. Several studies have dealt with the costs incurred when collecting the labeled training data needed for supervised learning. For example, Morales et al. [28] employed transfer learning to reuse kernels in convolutional neural networks (CNNs) across different domains. Rueda et al. [29] designed a $\mathrm{CNN}$ with parallel branches, with a branch processing data from each inertial sensor, and confirmed that the proposed network outperformed a baseline $\mathrm{CNN}$ when using a small amount of sensor data from the logistics domain. Maekawa et al. [26] reused labeled sensor data from source users with similar physical traits to those of a target user to train the target user's activity model. Work has also been done on using unsupervised learning to accomplish activity recognition, such as in Huynh et al. [9] where they clustered activity data in an unsupervised manner using topic models. Ye et al. [39] transfered activity labels across different partially labeled datasets from smart home environments that had distributed sensors. They leveraged home ontologies that describe the semantic relationships between sensor-enabled objects/places to determine the semantic similarities between distributed sensors in different environments, which are used to find sensor data patterns in different environments representing the same activity. Sanabria et al. [33] also employed home ontologies and variational autoencoders to achieve unsupervised domain adaptation to align the feature spaces of different environments. Krüger et al. [16] applied computational state space models (CSSMs), which have an ability to replace training data with prior knowledge, to human activity recognition. The authors confirmed that the CSSMs outperformed a training-based model (HMM) when the amount of information from training data is limited. Yordanova [40] focused on structured behavior such as cooking and automatically constructed a knowledge-based behavior model from instruction text that was used as a Planning Domain Definition Language (PDDL).

Several activity and gesture recognition studies have extracted motifs in an unsupervised manner. Minnen et al. [27] first found motif seeds using a minimum description length (MDL) criterion and then refined the motif seeds by splitting, merging, and extending the motifs. The MDL criterion permitted them to find motifs that were as long as possible and had high intra-motif similarity. They employed left-to-right HMMs to detect the occurrences of the motifs. Berlin et al. [7] recognized leisure activities by employing useful motifs in acceleration signals. They selected motifs that occurred at least two times and had a minimum length. In contrast to these studies, our study finds motifs useful for tracking repetitive works.

Techniques for recognizing and supporting factory work using sensor technologies have been actively studied $[1,3-5,13]$ due to the recent growing interest in Industry 4.0 and smart manufacturing $[6,20,30]$. For example, Koskimäki et al. [15] analyzed sensor data from a wrist-worn inertial sensor devices to ensure that all necessary operations were performed. They recognized activities such as hammering and screwing using a $k \mathrm{NN}$ search. In addition, Ward et al. [37] recognized woodworking activities by analyzing acceleration and audio data from a wrist-worn device using HMMs and a linear discriminative classifier. Stiefmeier et al. [35] analyzed assembly work on automobiles using inertial sensors attached to several locations on workers' bodies, such as the upper and lower arms. They classified sensor data segments by using the computed distance between the collected segments and sensor data templates prepared in advance. Stiefmeier et al. [34] also analyzed work on bicycle repair by recognizing motion sensors and ultrasonic hand tracking employing HMMs. All of these factory activity recognition studies relied on supervised machine learning and so required the creation of labeled training data.

In order to cope with issues encountered in supervised learning, unsupervised factory activity recognition has been studied recently in the ubicomp community. Maekawa et al. [25] measured the duration of each work period on a production line in an unsupervised manner. Their method tracked a motif that appears only once in each work period using a particle filter to estimate the duration of the work periods. Xia et al. [38] estimated the starting and ending times of each operation based on the tracked motif with the help of information derived 
from process instructions. However, as mentioned in the introduction section, these methods are vulnerable to outlier activities, which usually exist in factory work. In contrast, our method can robustly estimate the starting and ending times of each operation by leveraging two types of motifs, as was mentioned in the introduction section.

\section{ROBUST UNSUPERVISED FACTORY ACTIVITY RECOGNITION}

\subsection{Preliminaries}

3.1.1 Sensor Data Collection. This study assumes that workers are wearing a body-worn accelerometer. In our experiment, each worker wore a smartwatch on his/her right wrist, with the smartwatch's accelerometers collecting three-axis acceleration data at a sampling rate of $60 \mathrm{~Hz}$.

3.1.2 Process Instructions and Work Models. Process instructions are documents prepared in advance by a line manager for each work process to be performed by a worker. They specify the flow of operations performed during a work period, e.g., (1) place a board on the workbench, (2) remove film from the board, and (3) screw in parts, with some work periods containing optional operations, e.g., (4) pack accumulated boards into a box once 20 have been completed. These instructions also specify the standard duration for each operation. While the goal of this study is to estimate the start time of each operation in an unsupervised manner, it is impossible to determine which activity (operation) corresponds to each start time without prior information of the activities. In this study, we acquire the information from an existing process instruction document, and attempt to recognize the operations using only this document's information and unlabeled sensor data.

Using these process instructions, we can construct a work model that encapsulates key information from the instructions. This work model is represented by a tree-structure that describes the flow of operations, where the root node corresponds to the first operation in a work period with one node in the tree for every possible operation, as shown in Figure 3. Optional operations are represented in the tree by assigning multiple child nodes to the node immediately preceding the optional operation, with one branch of the split leading to the operation that normally follows and the other branch leading to the optional operation, e.g., the branch before the optional "Boxing" operation in Figure 3. Each node contains information about the expected duration of its corresponding operation, which is modeled as a log-normal distribution in this study.

Because this work model describes the order of operations and the duration of each operation, it is used to estimate the start time of the next operation in our method. While Xia et al. [38] used the semantics of the operations acquired from the descriptions of the operations, that study revealed that this information did not contribute much to the method's accuracy and incorporating this information required high computation costs when processing it in conjunction with the sensor data. Therefore, this study does not use that semantic information. Because our work model extracts only simple information, i.e., the flow of operations, from the process instruction document, it takes only a few minutes to construct the work model.

The number of leaf nodes in the tree corresponds to the total number of possible patterns of standard durations in the work period. In the example tree in Figure 3, there are two possible patterns of standard durations, due to the presence of the optional operation "Boxing." The overall standard lead time for the $i$-th pattern is computed by summing the standard durations of all nodes from the root node to the $i$-th leaf node in the tree, which is then used to track period motifs.

\subsection{Overview}

Figure 4 shows an overview of the proposed method. It is mainly composed of four procedures: (i) Discovering motifs, (ii) Building motif temporal structure, (iii) Tracking period motifs, (iv) Tracking operation start times. When discovering motifs, we first preprocess the data and then find period and action motifs within an initial segment of the sensor data (i.e., the first $t_{d m}$ minutes). We then build a model that describes the temporal structure 


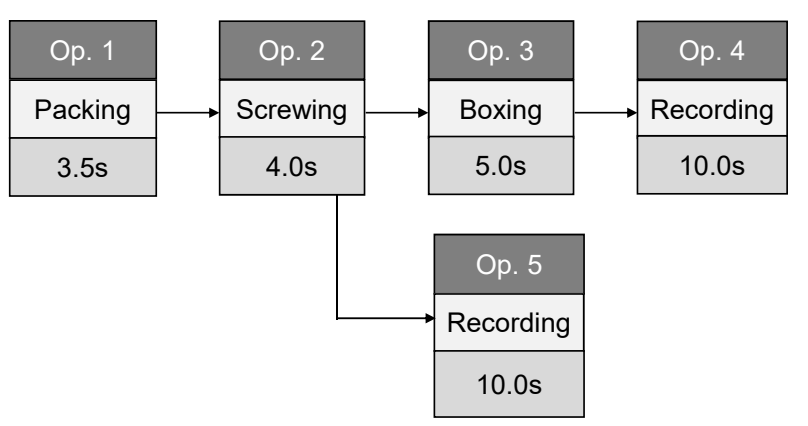

Fig. 3. Example flow of operations in the tree structure. The standard duration of each operation is shown at the bottom.

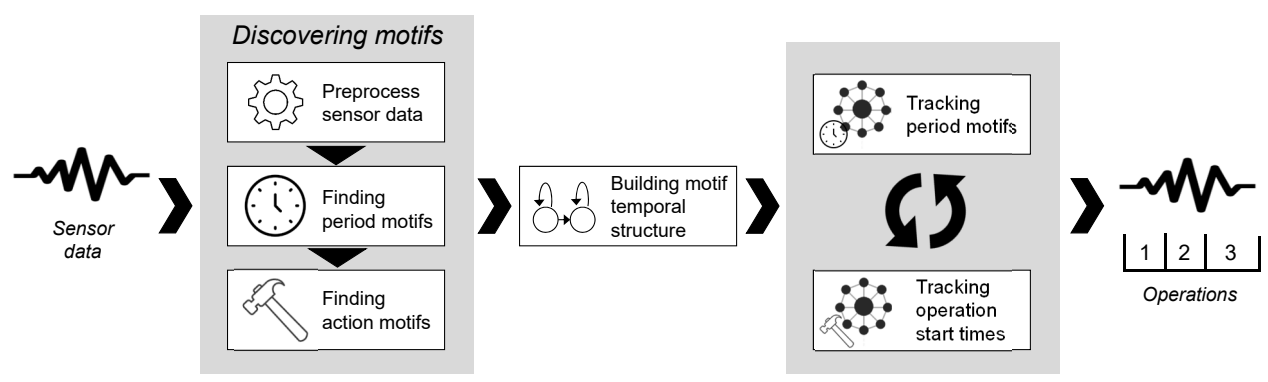

Fig. 4. Overview of the proposed method

Table 1. List of the symbols used in the following descriptions

\begin{tabular}{|c|c|} 
Parameter & Description \\
\hline \hline$t_{d m}$ & the duration of initial sensor data used for finding motif candidates \\
\hline$t_{i n i t}$ & the duration of initial sensor data used for calculating scores in motif selection \\
\hline$l_{m}$ & window length of motif, which is the shortest standard duration of operations in the instructions \\
\hline $\mathrm{S}$ & time-series of similarity values for a motif \\
\hline$k_{p}$ & the number of period motifs \\
\hline$k_{a}$ & the number of action motifs \\
\hline$t h_{p m}$ & similarity threshold used when detecting a period motif \\
\hline$d_{f}$ & a hyperparameter defining the possible minimum lead time $\left(0<d_{f}<1\right)$ \\
\hline$t_{o l}$ & an estimate of the duration of an outlier existing between two period motifs \\
\hline $\mathrm{C}$ & a set of trend changes \\
\hline
\end{tabular}

of the occurrences of the period motifs within that initial data segment. Finally, we run particle filters to track the period motifs through all work periods and combine the results from those particle filters with information about the action motifs in order to run a secondary particle filter that tracks the operation start times. Note that these particle filters are based on the work model and that our method does not require any labeled training data. Table 1 lists the symbols used in the following subsections. 


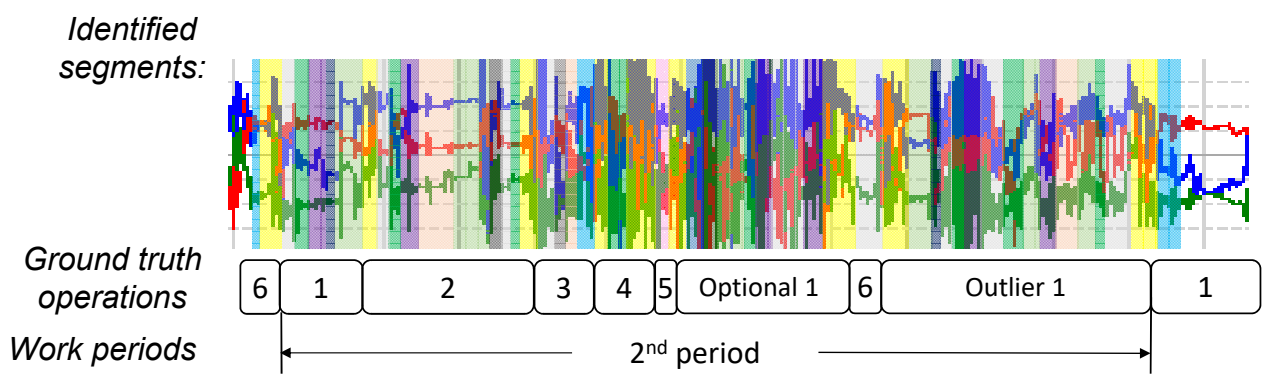

Fig. 5. Example segmentation results for acceleration data when using HDP-HMM to segment the data in an unsupervised manner.

\subsection{Discovering Motifs}

3.3.1 Preprocessing Sensor Data. In order to reduce computation costs, we first symbolize the input acceleration data based on previous factory activity recognition methods [25, 38]. In brief, we convert each of downsampled numerical acceleration values into a symbol based on the value range associated with each symbol (see the appendix section for more details). We then segment the sensor data to identify the likely starting locations for operations using HMMs (HDP-HMM; see the appendix section). The segmentation boundaries in the results output by HDP-HMM give us the locations of trend changes in the sensor data. These detected trend changes are then used to estimate the start times of operations in the tracking phase. Figure 5 shows example output from this segmentation method when applied to acceleration data collected from a factory worker. The overlaid color rectangles show the segments generated by this method. The numbered rectangles below the data represent the ground truth operations for the data. Although this segmentation method resulted in many false positives for operation start times, the number of false negatives is generally low. Therefore, we can use this segmentation method to generate candidates for operation start times, but they will require further refinement by later processes.

3.3.2 Finding Period Motifs. We start by finding period (and action) motifs within the first $t_{d m}$ minutes of sensor data starting from the beginning of collection. Having already preprocessed the data as described above, we randomly extract candidate period motifs with a length of $l_{m}$ from the first $t_{\text {init }}$ minutes of the sensor data, with $t_{\text {init }}<t_{d m}$. For each candidate period motif, we then compute a time series of similarity values. As shown in Figure 19 (b, c), we compute the similarity between each candidate period motif and consecutive data segments in the first $t_{d m}$ minutes of sensor data using a sliding window, which results in a new time series $\mathrm{S}$ of similarity values for the first $t_{d m}$ minutes of data. Note that the similarity metric is computed based on the Levenshtein distances between segments of symbolized data. Using the similarity time series S, we are now able to choose good period motifs from amongst the candidates. Figure 6 shows two examples of such similarity time series, with (a) showing the raw acceleration data and (b) and (c) each showing the similarity times series for a different candidate motif. Figure 6 (b) shows an example of a good candidate for a period motif, with the location of the motif's template indicated by a star. Meanwhile, Figure 6 (c) shows an example of a bad candidate for a period motif, with the template again indicated by a star. In the case of Figure 6 (b), we can find large peaks in the similarity values at the end of each work period, with a clear differentiation between the values in those peaks and the similarity values in rest of the work periods. This means that the waveform of the candidate motif is dissimilar to unrelated segments and appears consistently throughout the periods. In contrast, in Figure 6 (c), the differences between the similarity values at peaks and at other times are relatively small, making it difficult to detect occurrences of the motif. As this example shows, candidates that yield clear peaks should be selected. 

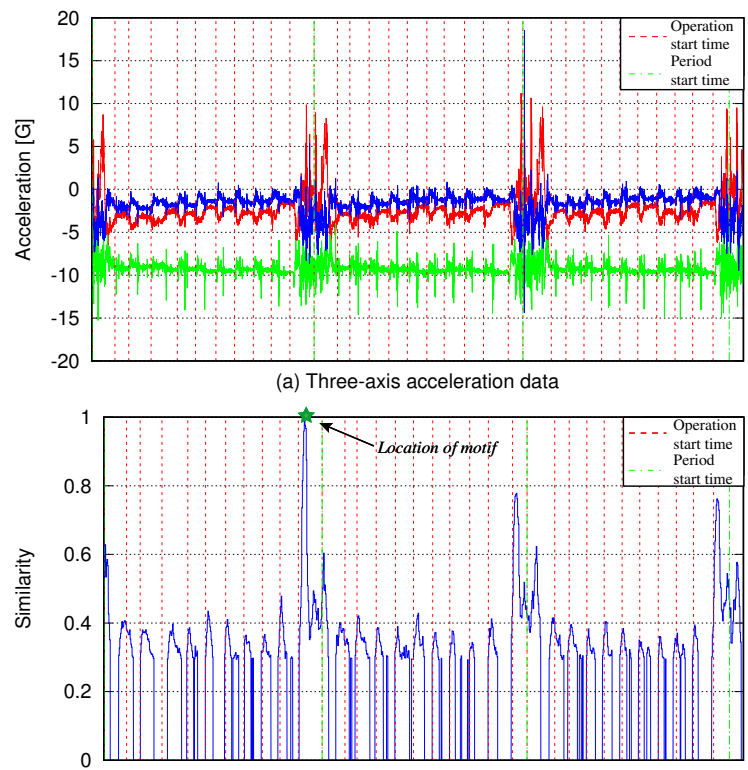

(b) Time-series of similarities of an example period motif 1

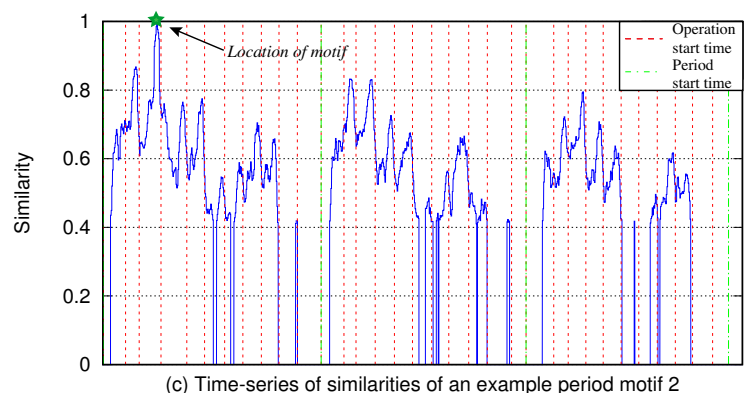

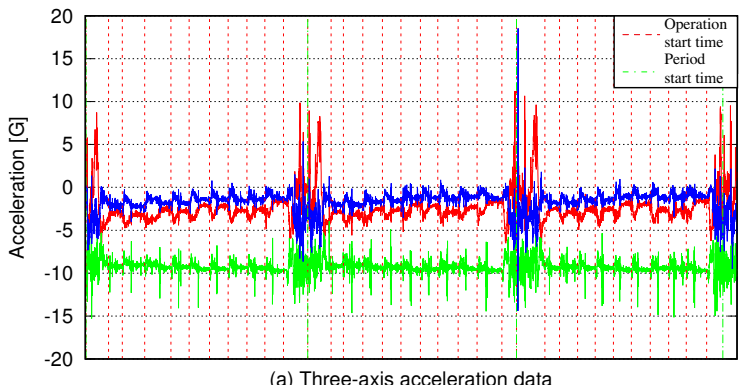

(a) Three-axis acceleration data

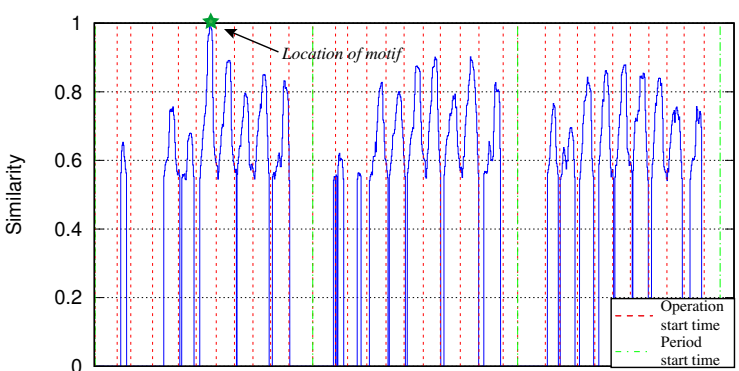

(b) Time-series of similarities of an example action motif 1

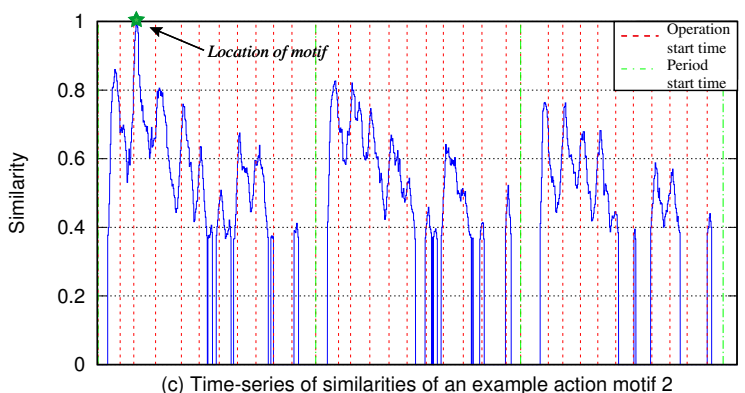

(c) Time-series of similarities of an example action motif 2

Fig. 6. Comparing the similarity time series for candidate pe- Fig. 7. Comparing the similarity time series for candidate acriod motifs. (a) The raw three-axis acceleration data. (b) The tion motifs. (a) The raw three-axis acceleration data. (b) The similarity time series for a good candidate for a period motif. similarity time series for a good candidate for an action motif. (c) The similarity time series for a bad candidate for a period (c) The similarity time series for a bad candidate for an action motif. The vertical dotted green lines show the start times for motif. The vertical dotted green lines show the start times for work periods, i.e., start time of the first operation. The vertical work periods, i.e., start time of the first operation. The vertical dotted red lines show the start time of each operation (except dotted red lines show the start time of each operation (except the first operation). The locations of the motif templates are the first operation). The locations of the motif templates are indicated with stars. indicated with stars.

We select the top- $k_{p}$ best period motifs from amongst the candidates using a combination of the following scores: (1) As shown in Figure 6 (b, c), the similarity values at peaks should be much larger than those at other times in the periods. Therefore, we compute the first score based on the difference between the peak similarity value and similarity values at the other time slices. (2) In addition to the above score, we simply use the similarity value of the candidate motif as the second score. For the complete equations of the above scores, see the appendix section. 
3.3.3 Finding Action Motifs. Just as with period motifs, we start our process of selecting good action motifs by first extracting candidate motifs and then computing a similarity time series $\mathbf{S}$ for each candidate using the first $t_{d m}$ minutes of sensor data. Figure 7 shows two examples of such similarity time series, with (a) showing the raw acceleration data and (b) and (c) each showing the similarity times series for a different candidate motif, with (b) showing an example of a good candidate and (c) showing an example of a bad candidate. In Figure 7 (b), the motif corresponds to a screwing action and the presence of clear peaks that repeat within each work period make it a good candidate. The thin peaks shown in this example are indicative of a good candidate for an action motif, as they make it easier to differentiate the peaks from background noise. In contrast, in Figure 7 (c), there is not a clear differentiation between similarity values at the peaks versus at other times in the work period, indicating that the candidate motif is not unique and is therefore hard to detect. Furthermore, the time series in Figure 7 (c) varies greatly from one work period to the next, indicating that the motif candidate yields an unstable similarity time series. In contrast, the time series in Figure 7 (b) is much more stable across work periods.

In order to find good action motifs such as the example shown in Figure 7 (b), we select the top- $k_{a}$ best action motifs from the candidates as follows. As shown in Figure 7 (b), the similarity values at occurrences of an action motif should be high and easily differentiated from the similarity values from other unrelated data segments. Therefore, we calculate the score as the difference between the mean similarity values of peaks and that of the other time slices. For the complete equations of the above score, see the appendix section.

\subsection{Building Motif Temporal Structure}

Using the processes described in the previous subsection, we are able to select good candidates for period motifs (and action motifs). Here we build a model of the motifs' temporal structure $(\mathcal{T})$ that captures the temporal distances between pairs of period motifs. This model represents the temporal structure using lists of observed temporal distances between the nearest occurrences of each of the motifs. Each list is initialized based on the relationship between the original data segments for the corresponding motifs in the first $t_{\text {init }}$ minutes of sensor data. Although each list contains only a single value upon initialization, the lists will be updated during the following procedures, enabling us to accurately model the temporal distances between period motifs. Given a temporal structure model $\mathcal{T}, \mathcal{T}\left(m_{i}, m_{j}\right)$ outputs a single value calculated as the average value for the list of temporal distances between the pair of motifs $m_{i}$ and $m_{j}$, which is used in the tracking procedure.

\subsection{Tracking Period Motifs}

We track period motifs using particle filters, with a separate particle filter prepared for each motif. Note that of these particle filters, the one with the highest score is hereinafter referred to as the base particle filter, with the period motif tracked by this particle filter referred to as the base period motif. Because the base particle filter's results are more reliable than those from the other lower-scoring particle filters, its results can be used to correct the results for those other particle filters. This study employs particle filters to track period motifs (and operation start times) because the robustness and effectiveness of particle filters in unsupervised factory activity recognition were confirmed in previous studies $[25,38]$. In this study, we introduce multiple period motifs into the particle-filter-based activity recognition in order to achieve more robust tracking.

Particle filtering consists of three procedures: sampling, weighting, and resampling. During the sampling procedure, we generate new particles that represent predicted locations for the occurrence of a period motif in the $(i+1)$-th period based on its estimated occurrence in the $i$-th period, as shown in Figure 8 (a). In the weighting procedure, we calculate a score for each of those new particles based on the motif's similarity time series and the results from the other motifs' particle filters. In the resampling procedure, we probabilistically discard particles with low scores. We explain these procedures in detail below. 


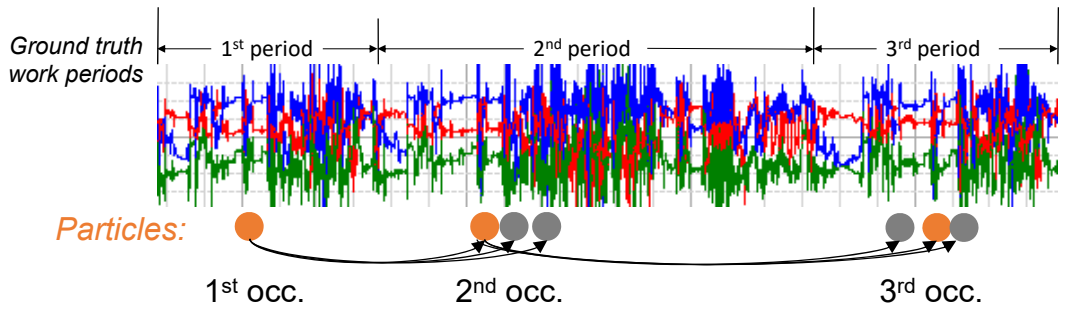

(a) Tracking occurrences of a period motif

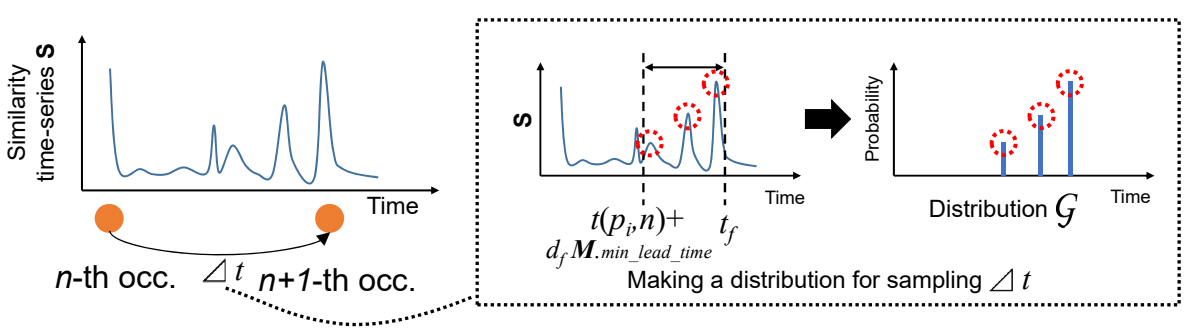

(b) Sampling for $n+1$-th occurrence

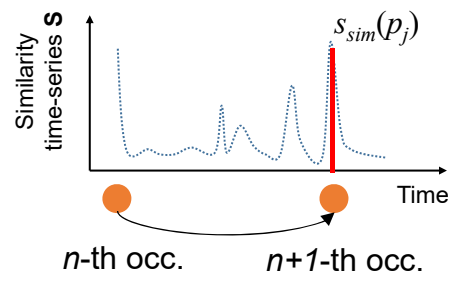

1) Similarity score

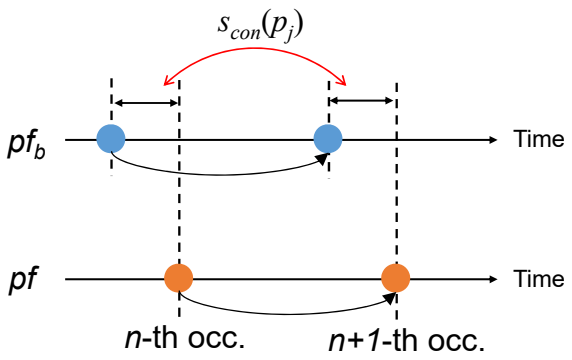

2) Consistency score

(c) Weighting for $n+1$-th occurrence

Fig. 8. Overview of the procedures used when tracking period motifs based on particle filters. (a) We start with an initial particle at the first occurrence of the period motif. We then sample several particles from this initial particle (three in this example), which are the initial estimates for the second occurrence of the motif. These sampled particles are then weighted and resampled (one in this example), leaving us with our most likely estimate(s) for this occurrence. The most likely estimates are then sampled to generate the estimates for the third occurrence of the motif. (b) During the sampling phase, new particles are generated at a distance of $\Delta t$ from the previous particle, with $\Delta t$ sampled from a distribution $\mathcal{G}$ that is based on the similarity time series $\mathrm{S}$ with the likelihood of sampling a particle at $t$ proportional to the similarity value at $t$. (c) The $n+1$-th occurrence of a particle is weighted using a combination of a similarity score and a consistency score. The similarity score is the similarity value at the $n+1$-th particle. The consistency score measures the consistency of the temporal distance between the $n+1$-th occurrence of the current motif $p f$ and the $n+1$-th occurrence of the base motif $p f_{b}$ when compared to their temporal distances in past work periods.

3.5.1 Initialization. When tracking a period motif, we start by creating a tree structure, which we will refer to as a history tree, that is used to track all the particles generated for that motif. We generate the first particle for 
that motif at the location of its first occurrence in the sensor data and place that particle in the tree as the tree's root node.

3.5.2 Sampling. We randomly sample subsequent occurrences for each particle based on $\mathrm{S}$ and the standard lead times from the work model $\mathcal{M}$, which is illustrated in Figure $8(\mathrm{~b})$. Assuming that $t\left(p_{i}, n\right)$ is the timestamp for a particle $p_{i}$ generated during the $n$-th iteration of sampling, then the timestamp for some new particle $p_{j}$ from among the $m$ particles generated from $p_{i}$ during the $n+1$-th iteration of sampling is determined using the following equation.

$$
t\left(p_{j}, n+1\right)=t\left(p_{i}, n\right)+\Delta t
$$

where $\Delta t$ is the estimated interval between $p_{i}$ and $p_{j}$ that is randomly sampled from a distribution $\mathcal{G}$ generated from $\mathrm{S}$ and standard lead times. $\mathcal{G}$ is a discrete distribution that describes possible locations of the $n+1$-th occurrence of the motif, which is illustrated in the right portion of Figure 8 (b) and formulated as follows.

$$
\mathcal{G}=\operatorname{Disc}\left(\operatorname{peak}\left(\mathrm{S}_{\left(t\left(p_{i}, n\right)+d_{f} \cdot \mathcal{M} . m_{i n} \text { lead_time }_{f}\right)}\right)\right),
$$

where $t_{f}$ is a time when S first exceeds $t h_{p m}$ (see Section 6.2) after $t\left(p_{i}, n\right)+d_{f} \cdot \mathcal{M}$.min_lead_time plus a margin,

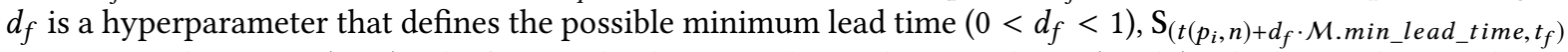
is a segment between $t\left(p_{i}, n\right)+d_{f}$. M.min_lead_time and $t_{f}$ within S, and DISc(peaks) calculates a discrete distribution where the probability of sampling at a peak is proportional to the value at that peak and the probabilities at time slices other than the peaks are zero.

Each of the $m$ particles generated from $p_{i}$ are then our estimates for the possible $n+1$-th occurrences of the motif, with each new particle $p_{j}$ being stored as a child node of $p_{i}$ in the history tree.

3.5.3 Weighting. After having sampled new particles based on the discrete distribution, we then calculate a score for each new particle $p_{j}$ using a combination of the following scores, which are illustrated in Figure 8 (c): Similarity score: The similarity value at $t\left(p_{j}, n+1\right)$ should be high if the particle is a good candidate. Therefore, we simply use the similarity value as a score. Consistency score: The temporal distances between the occurrences of a period motif and the base period motif should be consistent throughout work periods. Therefore, we compute the consistency of the temporal distances in the period of interest. For more details about score calculation, see the appendix section.

3.5.4 Resampling. Here we employ roulette wheel selection to probabilistically resample the generated particles. In roulette wheel selection, each particle is randomly selected with a probability proportional to the score assigned to that particle during the weighting phase. This method enables us to select the high-scoring particles with higher probability and discard the remaining particles. The discarded particles are removed from the history tree. The posterior estimate of the occurrence time for the motif of interest o(pf.motif, $n+1)$ is the weighted average of the resampled particles, with their scores used as the weights.

Finally, we update the temporal structure model for each pair of motifs by appending the temporal distance between their most recent occurrences (posterior estimates) to the list.

\subsection{Tracking Operation Start Times}

The particle filters for period motifs output timestamps for the occurrences of the motifs. In addition, we have timestamps for trend changes $\mathrm{C}$ (candidates of operation start times) that are output by HDP-HMM. Furthermore, we have information about the standard duration for each operation which is described in the work model $\mathcal{M}$. We now use this information as input to a particle filter that produces our estimates for the most likely start times for all operations in a work period. We start by initializing this particle filter by generating initial particles as candidates for the start time of the work period (i.e., the start time of the first operation). We then track each of these particles to identify the start times of the following operations in the work period by iterating through 


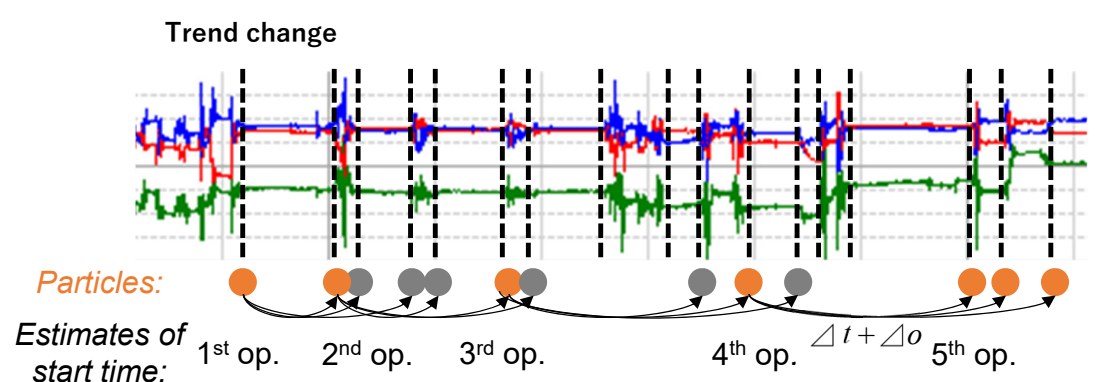

(a) Tracking starting times of operations

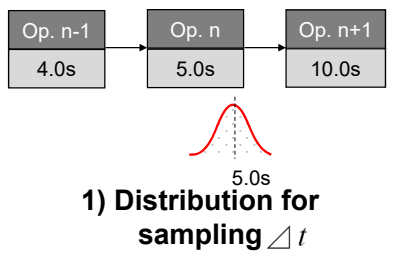

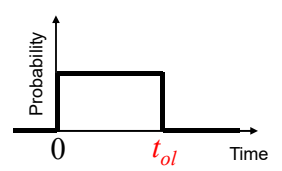

2) Distribution for sampling $\triangle o$

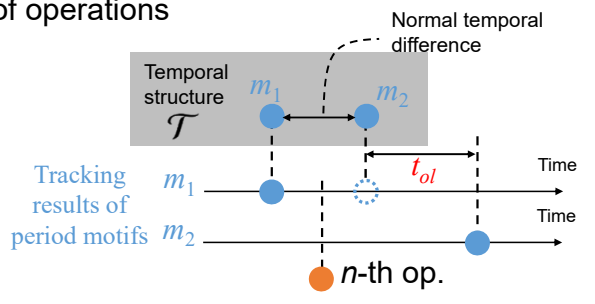

3) Determining $t_{o l}$

(b) Sampling for $n+1$-th operation

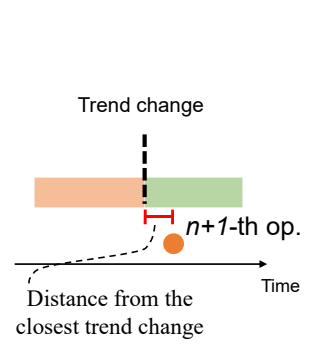

1) Trend-based score

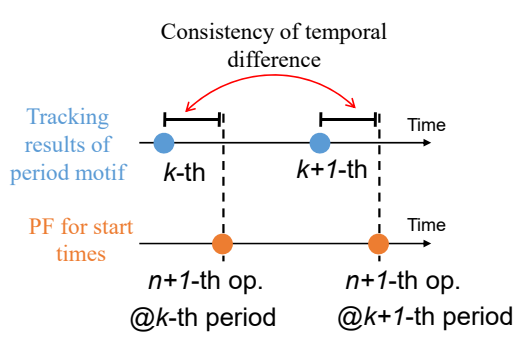

2) Period-motif score

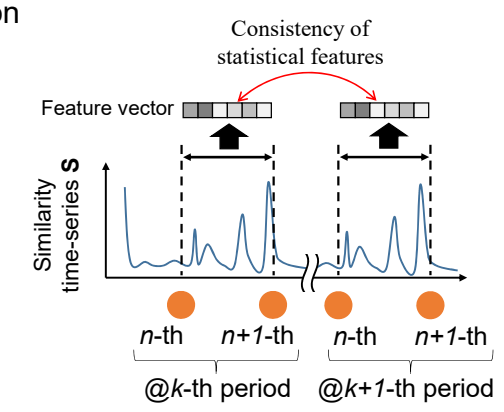

3) Action-motif score

\section{(c) Weighting for $n+1$-th operation}

Fig. 9. Overview of the procedures used when tracking operation start times based on particle filters. (a) Starting from an initial particle representing our estimated start time for the first operation, we sample several particles (three in this example), which are the initial estimates for the start time of the second operation. These sampled particles are then weighted and resampled, leaving us with our most likely estimate(s) for the start time of the second operation (one in this example). (b) During the sampling phase, the temporal distance between the particles for the $n$-th and $n+1$-th operations is $\Delta t+\Delta o$. $\Delta t$ is sampled from a distribution of the duration of the $n$-th operation described in $\mathcal{M}$. $\Delta o$ is sampled from a uniform distribution over $\left[0, t_{o l}\right] . t_{o l}$ is an estimate of the duration of outliers, which is calculated as the extended time of the temporal distance between period motifs $m_{1}$ and $m_{2}$ compared to that in the temporal structure model $\mathcal{T} . m_{1}$ and $m_{2}$ are motifs just before/after the start time of the $n$-th operation. (c) Weighting phase of the particle filter for a particle corresponding to the start time of the $n+1$-th operation. The trend-based score for the particle is calculated based on the temporal distance from the closet trend change. The period-motif score for the particle is calculated based on the consistency of the temporal distances from adjacent period motifs. The action-motif score for the particle is calculated based on the consistency of statistical features extracted from the similarity time series corresponding to the estimated $n$-th operation. 
the particle filter's three phases of sampling, weighting, and resampling as shown in Figure 9 (a). Furthermore, because our method tracks the start times of the operations in the order that they are described in the work model, our method can determine the class label of a segment between two adjacent start times in the unsupervised manner. We explain each of these procedures in detail below.

3.6.1 Initialization. As shown in Figure 2, the start time of the first work period exists somewhere before the first occurrence of the first motif $\left(m_{11}\right)$. Therefore, we can generate initial particles at each timestamp from C that fall between the starting time of the sensor data and the first occurrence of the first motif, which become our candidates for the start time of the first operation in the first work period. We track the estimated operation start times using a tree structure, with a tree created for each initial particle with that initial particle set as the tree's root node.

3.6.2 Sampling. As shown in Figure $9(\mathrm{a}, \mathrm{b})$, with each iteration we sample new particles from each existing particle that represent estimates for the start time for the following operation. Assuming that $t\left(p_{i}, n, k\right)$ is the timestamp for an existing particle $p_{i}$ that represents an estimate of the start time of the $n$-th operation in the $k$-th work period, then the timestamp $t\left(p_{j}, n+1, k\right)$ for a new particle $p_{j}$ for the $n+1$-th operation is sampled from $p_{i}$ as follows:

$$
t\left(p_{j}, n+1, k\right)=t\left(p_{i}, n, k\right)+\Delta t+\Delta o,
$$

where $\Delta t$ is randomly sampled based on the duration-time distribution in the work model tree that corresponds to the current node (i.e., the $n$-th operation's node). $\Delta o$ represents estimates for the duration of an outlier that possibly exists between the $n$-th and $n+1$-th operations and is randomly sampled from a uniform distribution over $\left[0, t_{o l}\right]$, where $t_{o l}$ is an estimate of the duration of any outlier segments existing between the occurrence of period motif $m_{1}$ just before $t\left(p_{i}, n, k\right)$ and the occurrence of another period motif $m_{2}$ just after $t\left(p_{i}, n, k\right)+\Delta t$. When the temporal distance between the two occurrences, i.e., $o\left(m_{2}, k\right)-o\left(m_{1}, k\right)$, is much larger than $\mathcal{T}\left(m_{2}, m_{1}\right)$ as shown in the right part of Figure 9 (b), we can assume that there are some outlier segments between the two occurrences. Therefore, $t_{o l}$ is calculated as follows.

$$
t_{o l}= \begin{cases}\left(o\left(m_{2}, k\right)-o\left(m_{1}, k\right)\right)-\mathcal{T}\left(m_{2}, m_{1}\right) & \left(\left(o\left(m_{2}, k\right)-o\left(m_{1}, k\right)\right)-\mathcal{T}\left(m_{2}, m_{1}\right)>t h_{o l}\right) \\ 0 & \text { (otherwise })\end{cases}
$$

Each new particle $p_{j}$ is then stored as a child node of $p_{i}$ in the history tree for operation start times.

3.6.3 Weighting. As shown in Figure 9 (c), we compute a weight for each particle generated in the sampling phase using a combination of three scores: a trend-based score, a period-motif score, and an action-motif score. These scores are calculated as follows: Trend-based score: As depicted in Figure 5, operation start times usually exist close to trend changes in the sensor data. Therefore, we calculate the temporal distance between the timestamp of a particle and the timestamp of the closest trend change. Period-motif score: The temporal distance between the start time of the $n+1$-th operation and an adjacent period motif should be consistent throughout work periods. Therefore, we calculate the consistency of the temporal distances within the period of interest. Action-motif score: A similarity time series is given for each action motif, which represents the occurrences of that action motif. The similarity segments corresponding to a given operation should be similar with each other across work periods. Therefore, we calculate the consistency of each operations segments within the period of interest. To reduce the computation costs related to the similarity calculation, we extract a feature vector consisting of simple statistical features such as the standard deviation from each segment, and then calculate the inverse of the Euclidean distance between the vectors. For more detail about the score calculation, see the appendix section. 
3.6.4 Resampling. First, we probabilistically resample the particles using roulette wheel selection with each particle resampled with a probability proportional to its weight that was assigned above, with any discarded particles removed from the history trees. We next need to determine which operation to use when estimating the following operation start time, since the value for $\Delta t$ will be set based on that operation's duration-time distribution in the work model. In general, we can simply use the following operation in the work model, unless there is a branch in the model, in which case we randomly select the branch to follow. Each of the remaining particles is then used to generate new particles during the sampling phase of the next iteration.

Note that, as shown in Figure 2, the end time of the first work period exists somewhere between the first occurrence of the last motif $\left(m_{31}\right)$ and the second occurrence of the first motif $\left(m_{12}\right)$. Therefore, we delete particles that do not fall between the $n$-th occurrence of the last period motif and the $n+1$-th occurrence of the first period motif at the end of each period.

We iterate through these processes of sampling, weighting, and resampling until reaching the end of each period, with the most likely overall sequence of start times finally selected based on the particle that has the highest weight at the end of the period.

3.6.5 Post-processing. During post-processing, we examine each operation that was determined to contain outliers in order to identify which segments in that operation correspond to outlier activity and which correspond to non-outlier activity, i.e., the actual operation. We do this by analyzing the operation using a sliding window with length set equal to the standard duration of the operation, extracting features from the sliding windows using the features described in the section on action motifs. For each window, we then compute the average Euclidean distance between that window's feature vector and feature vectors extracted from the current operation when it occurred without outliers in prior work periods. The time window with the smallest averaged distance then corresponds to the non-outlier segment, and all remaining segments are regarded as being outlier segments.

\section{EVALUATION}

\subsection{Dataset}

We evaluated the proposed method using six datasets collected from workers in real factories. Table 2 shows an overview of all the datasets. Note that since each work process is performed by a different worker, the total number of workers is six. The number of standard operations included in a work process is shown in the "\# operations" row, with the number of optional operations shown in parentheses, e.g., work process B consists of 6 operations plus 1 optional operation. The standard duration for one work period is shown in the "standard duration" row, with the standard duration when optional operations are present shown in parentheses, e.g., the standard duration of work process B is normally 59 seconds and is 87 seconds when the optional operation is performed. The total number of work periods included in the observed sensor data for each work process is shown in the "periods" row. The total duration of the observed sensor data for each work process is shown in the "data duration" row. Note that the datasets used in this evaluation were collected in the same factories as datasets used in [38]. However, [38] did not use datasets containing outliers because that did not assume the presence of outlier data segments. In contrast, this study uses these datasets containing outliers, which are usually observed in actual factories. The total duration of the outlier activities in the observed sensor data for each work process is shown in the "outlier duration" row. The datasets were collected in two different factories (factories A and B), with the "factory" row identifying the factory in which each work process was conducted. An overview of each work process is shown in the "work overview" row. Work process A involves testing the functions of a circuit board. To test each board, the worker places the board on a dedicated table, operates several dials, and pushes buttons. Work process B involves checking and packing items. The worker checks the appearance of an item, attaches a label to the item, scans a bar-code on the label, bags the item, and packs the bag in a box. When the box is full, the worker arranges a new box. Work processes $\mathrm{C}$ and D involve checking products. The worker 
Table 2. Overview of our dataset

\begin{tabular}{|c||c|c|c|c|c|c|}
\hline & work A & B & C & D & E & F \\
\hline \hline \# operations & 8 & $6(1)$ & 7 & 7 & 11 & 11 \\
\hline standard duration [s] & 130 & $59(87)$ & 53 & 50 & 55 & 50 \\
\hline periods & 10 & 9 & 14 & 13 & 12 & 11 \\
\hline data duration [s] & 1440 & 614 & 778 & 632 & 676 & 674 \\
\hline outlier duration [s] & 0 & 76 & 51 & 14 & 0 & 79 \\
\hline factory & $\mathrm{A}$ & $\mathrm{A}$ & $\mathrm{A}$ & $\mathrm{B}$ & $\mathrm{B}$ & $\mathrm{B}$ \\
\hline work overview & $\begin{array}{c}\text { test and record } \\
\text { circuit board } \\
\text { information }\end{array}$ & $\begin{array}{c}\text { bag and box } \\
\text { circuit } \\
\text { boards }\end{array}$ & $\begin{array}{c}\text { check final } \\
\text { product and } \\
\text { record results }\end{array}$ & $\begin{array}{c}\text { check final } \\
\text { product and } \\
\text { record results }\end{array}$ & $\begin{array}{c}\text { install screws } \\
\text { on circuit } \\
\text { board }\end{array}$ & $\begin{array}{c}\text { install screws } \\
\text { on circuit } \\
\text { board }\end{array}$ \\
\hline
\end{tabular}

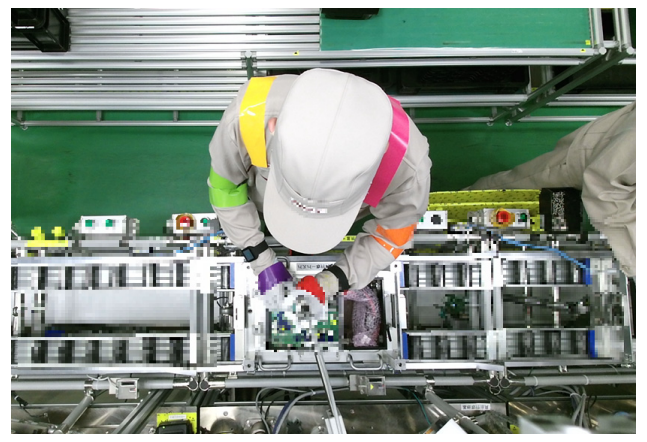

Fig. 10. Image from video captured in the factory

places a product on a dedicated table, scans a label on the product, measures the size of the product, checks the appearance, and boxes the product. Work processes $\mathrm{E}$ and $\mathrm{F}$ involve installing screws on a circuit board. The worker places parts on a board, installs eight screws to fasten the parts, and pushes several buttons to finalize the process.

The sensor data used in this study was captured at approximately $60 \mathrm{~Hz}$ using a Sony SmartWatch3 SWR50 attached to each worker's right wrist. The ground truth data needed for evaluation was recorded using video cameras, with Figure 10 showing an example of the images captured. The data was processed offline in our laboratory after data collection.

\subsection{Evaluation Methodology}

Our method outputs the start times for all operations in each work period, allowing us to classify each acceleration data point as belonging to an operation class. Therefore, we treat our results as classification results estimated per acceleration data point, allowing us to calculate the macro-averaged F-measure for our results. Because our method can also label outlier segments, an "outlier" class is included when calculating the F-measure.

To evaluate the effectiveness of the proposed method, we prepared the following methods.

- Proposed: This is the proposed method.

- Only-Base: The proposed method only using a single period motif (base period motif). 


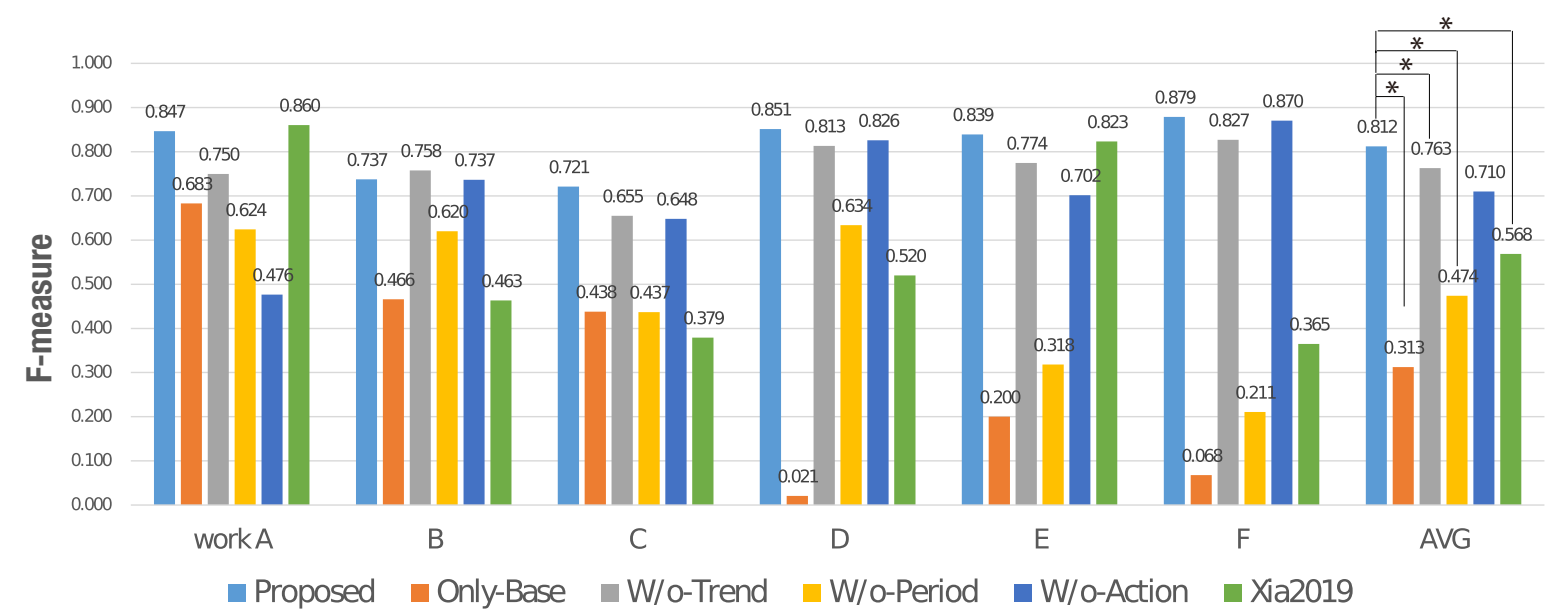

Fig. 11. F-measures of the methods. Paired t-tests were used to confirm any significant differences between the results for each method. ${ }^{*} p<0.05$.

- W/o-Trend: The proposed method without the use of the trend-based scores in the particle filters used for tracking start times.

- W/o-Period: The proposed method without the use of the period-motif scores in the particle filters used for tracking start times.

- W/o-Action: The proposed method without the use of the action-motif scores in the particle filters used for tracking start times.

- Xia2019: This is the method proposed from [38]. This method relies on a single motif and similarity calculation based on DTW. For more details, see [38].

\subsection{Results}

4.3.1 Recognition Accuracy. Figure 11 shows the classification accuracy for each method. Overall, the Proposed method achieved the highest average F-measure across all six work processes ( 0.81$)$. Even when only considering the work processes that contain outliers, the average F-measure for Proposed was still 0.80 . These results confirm the robustness of the proposed method when used on real-world factory work sensor data that contains outliers. In addition, the overall trends of the six methods for the precision and recall are similar to the trend of F-measure. Because the recognition errors are mainly caused by the temporal shifts of estimates from the ground truth, the precision, recall, and F-measure of a method have similar values.

Figures 12 and 13 show examples of output from the Proposed method. The individual operations are indicated by the colored bars, with the upper half of the bars indicating the ground truth segmentation and the lower half of the bars indicating the estimated segmentation output by Proposed. The numbers in the upper margin correspond to the operation number for each ground-truth segment. The tracking results for selected period motifs are also shown in the upper part of the figure. As shown in these figures, Proposed was able to successfully identify the occurrences of the outlier activities even though information about outliers was not given in advance. Figure 12 also provides a good example of the importance of using multiple period motifs. In this case, we can 


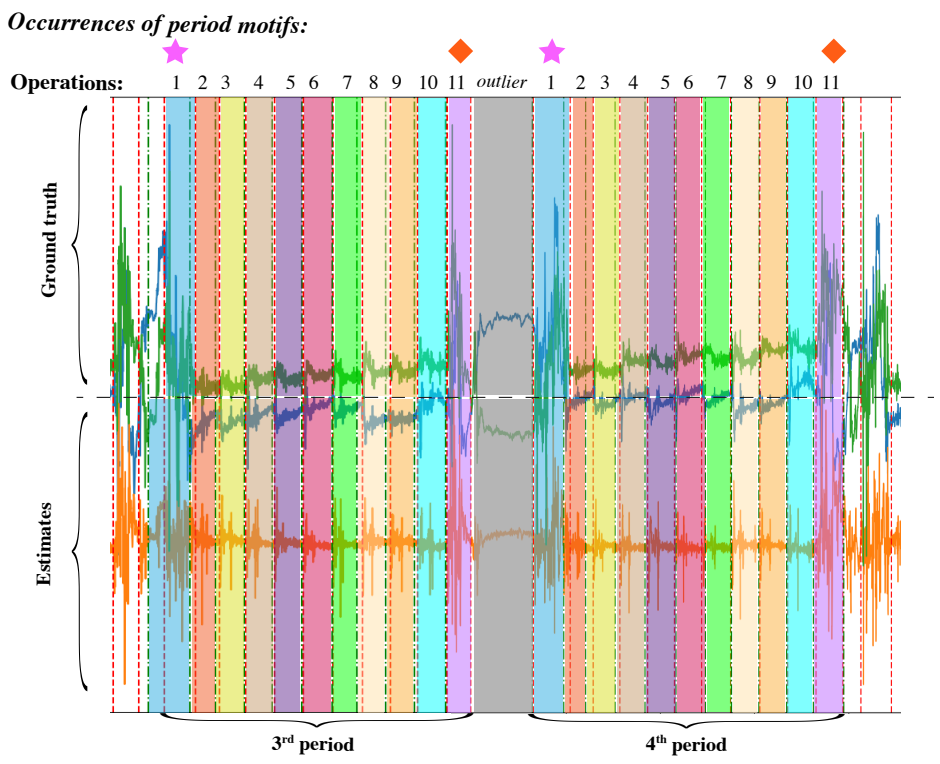

Fig. 12. Example output from Proposed for the third and fourth periods of work process $F$

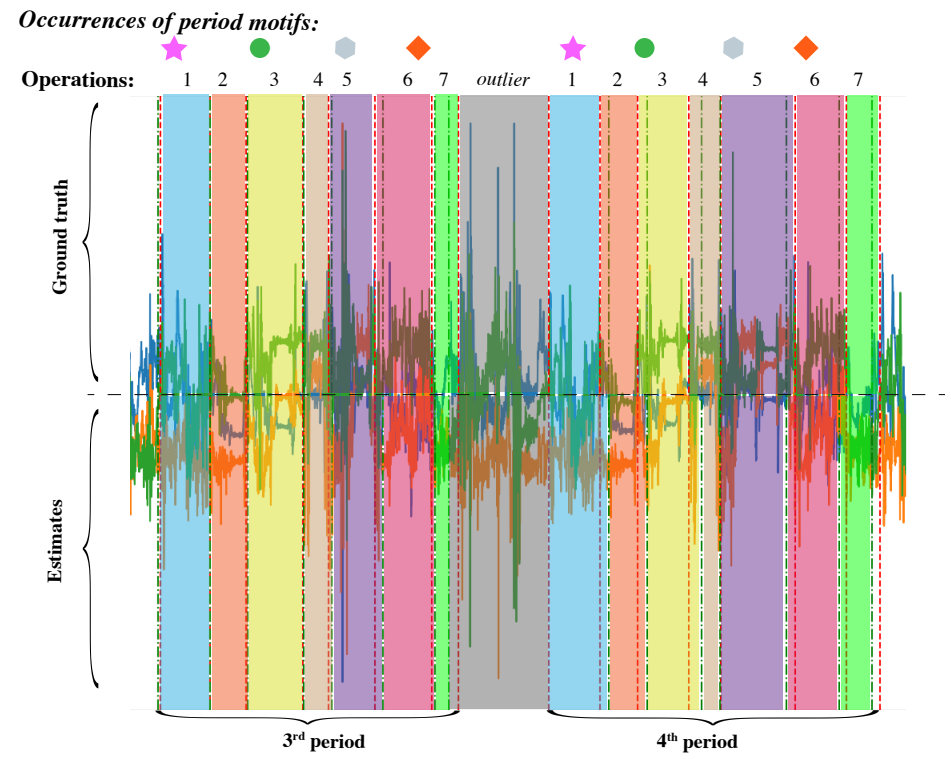

Fig. 13. Example output from Proposed for the third and fourth periods of work process D

see how that the period motifs located in the 1st and 11th operations effectively surrounded the outlier activity allowing Proposed to precisely locate its start and end times.

Proc. ACM Interact. Mob. Wearable Ubiquitous Technol., Vol. 4, No. 3, Article 97. Publication date: September 2020. 


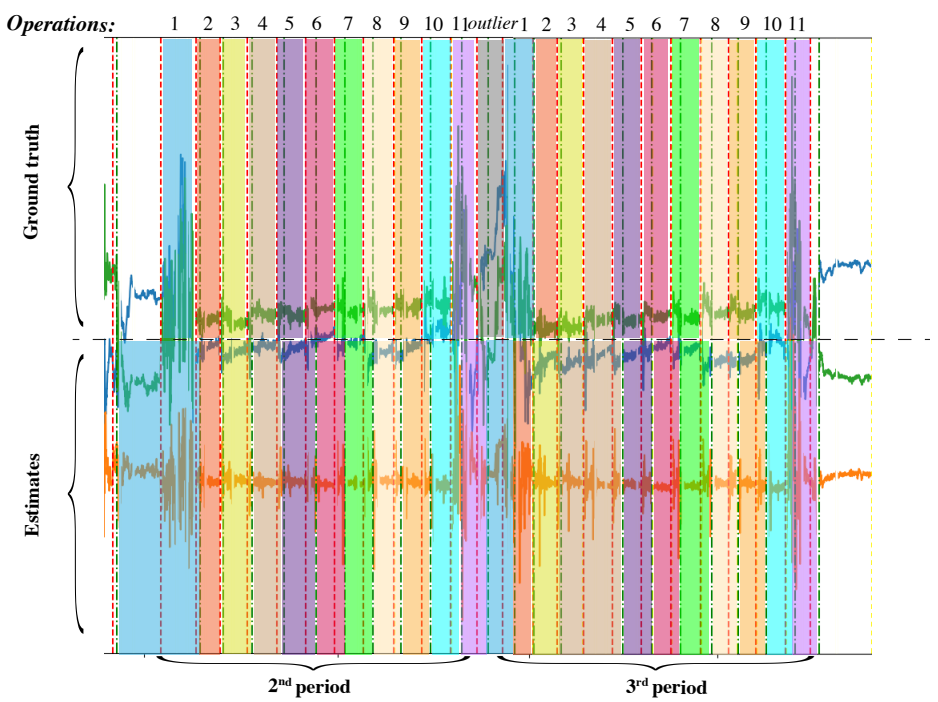

Fig. 14. Example output from Xia2019 for the second and third periods of work process $F$

Proposed did not achieve the highest F-measure for every work process, with the F-measure of Xia2019 higher than that of Proposed for a work process without outliers (work process A), despite performing significantly worse than Proposed overall. Because Xia2019 employs DTW to exactly match waveforms of an operation in different periods, Xia2019 achieved the high F-measures when outlier activities did not occur (work processes A and E). However, the use of DTW in Xia2019 significantly increases its computation costs when compared to Proposed, which is discussed in further detail below. Also of note are the poor results from the Xia2019 method in the work processes that contained outliers, again indicating the need for factory activity recognition methods to include some mechanism for handling outliers. For example, Figure 14 shows the output from Xia2019 when an outlier segment was present. Because Xia2019 could not detect the outlier segment, the estimated start times for the operations that occurred after the outlier segment were shifted forward.

4.3.2 Transitions in Recognition Accuracy. Figure 15 breaks down the average F-measures from Figure 11 into the individual F-measures for each work period. As shown in these results, the F-measures dropped during or after outlier activities in many cases, reducing the overall F-measures. However, in some cases Proposed could maintain a high F-measure even when outlier activities occurred (e.g., the 4th work period in work process D). As shown in Figure 15, the F-measures of Xia2019 dropped in the 4th period of work process C, which does not contain outliers. This is because the duration of one particular operation in that period was much longer than usual. While the durations of many operations in work process $\mathrm{C}$ were unstable, deteriorating the F-measures for all the methods, Proposed was able to maintain reasonable F-measures. Overall, Proposed maintained high Fmeasures even in the later work periods, as shown in Figure 15. This can be because Proposed robustly tracks the start times of operations by leveraging multiple period motifs. In contrast, the F-measures of Xia2019 gradually decreased in many cases.

4.3.3 Effect of Multiple Period Motifs. Figure 11 includes the results for the Only-Base method, which employs only a base period motif. As can be seen in these results, the F-measures of Only-Base are poor for many work processes, indicating that tracking multiple period motifs was an important aspect of Proposed. While the base 


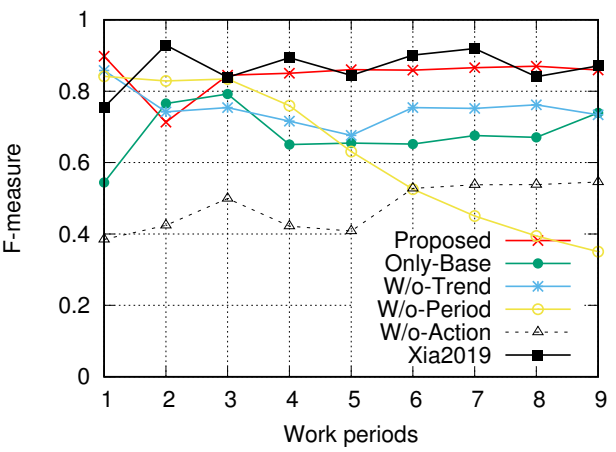

Results from work process A

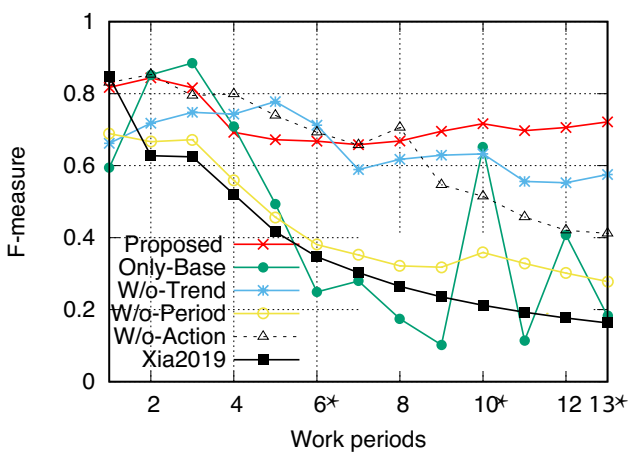

Results from work process C

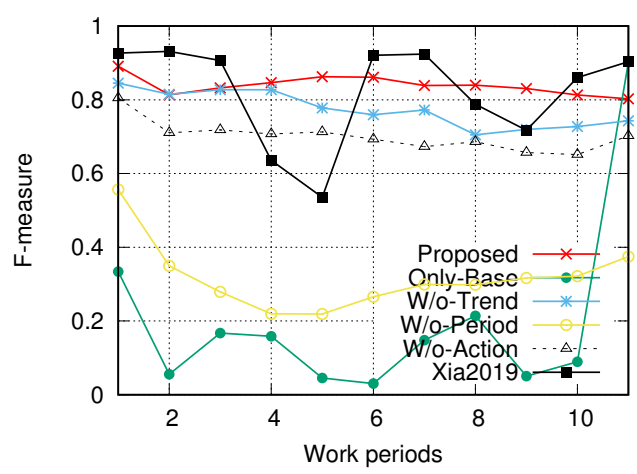

Results from work process E

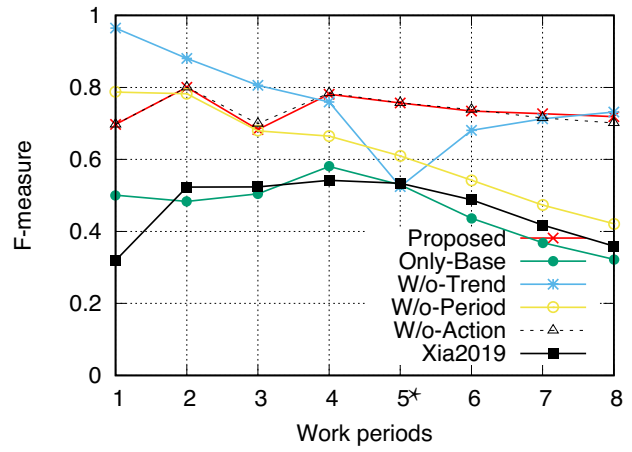

Results from work process B

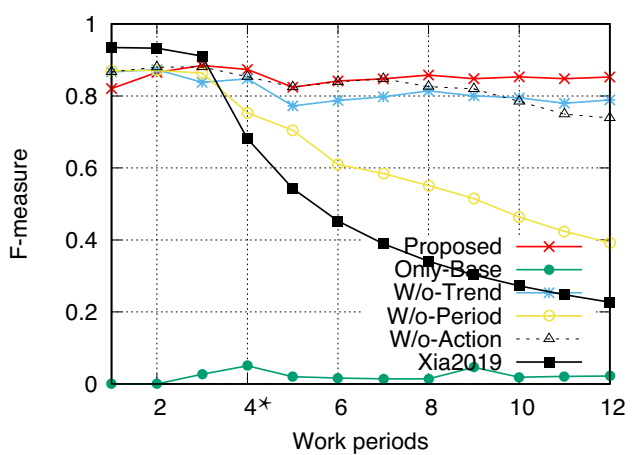

Results from work process D

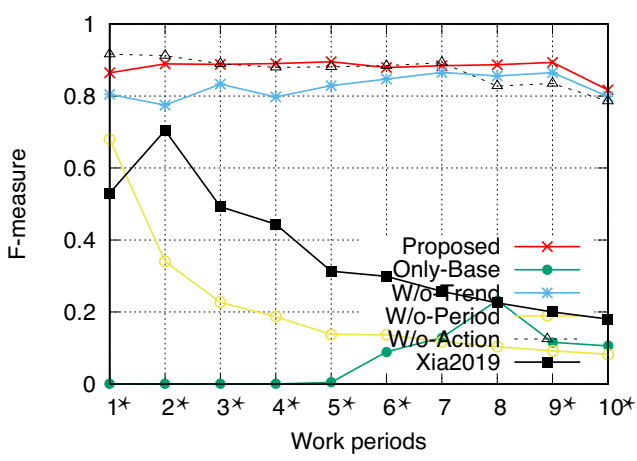

Results from work process $\mathrm{F}$

Fig. 15. Transitions in F-measures of each method. The $\left(^{*}\right)$ in the horizontal axis indicates periods with outliers.

particle filter is reliable, it is difficult to recognize the fine-grained structure of the work (i.e., start times of operations) using only a single period motif.

4.3.4 Effect of Trend-based Score. As can be seen in the W/o-Trend results in Figure 11, the F-measure of W/oTrend is higher for many work processes than those of W/o-Period and W/o-Action. This indicates that the 


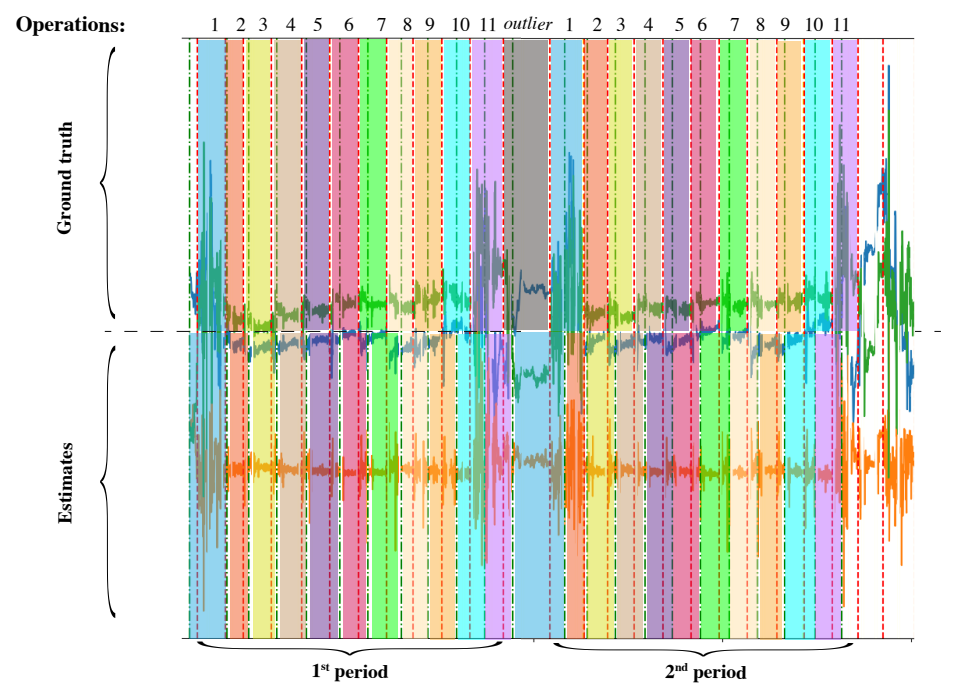

Fig. 16. Example output from W/o-Period for the first and second periods of work process $\mathrm{F}$

contribution of the trend-based score is lower than the contributions of the period-motif and action-motif scores. Note that the process of detecting trend changes is the most time-consuming part of the Proposed method, described in further detail below, indicating that eliminating this score is one possible way to further improve computation costs.

4.3.5 Effect of Period-motif Score. Figure 11 also shows the results for W/o-Period, which does not employ the period-motif score. The F-measures of W/o-Period are poor for many work processes, indicating that the temporal relationship between the occurrences of period motifs and operation start times was an important aspect of Proposed. Because Proposed tracked about 5 to 10 period motifs in comparison to the 6 to 11 operations per process, the density of these motifs allowed it to precisely detect the start times of operations. Figure 16 shows an example result for W/o-Period for work process F. Here, we can see that although W/o-Period employs the action-motif and trend-based scores, it still could not precisely identify the start times of the operations. A major factor in W/o-Period's poor performance was its inability to detect outlier segments, which can also be seen in Figure 16. These results indicate that the use of dense period motifs is integral to the proposed method's ability to detect outliers.

4.3.6 Effect of Action-motif Score. Looking at the W/o-Action results in Figure 11, we can confirm that the action-motif score was the second most important contributor among the three scores. Note that there was not a significant difference between the overall results for Proposed and W/o-Action because Proposed greatly outperformed W/o-Action only in work processes A and E. The F-measure for work process A for W/o-Action was poor because we had few period motifs in this work, indicating that action motifs played an important role in this work. Figure 17 shows an example result for W/o-Action for work process E. As shown in the figure, the estimated start times of operations in the second period are slightly shifted. In this case, our method only found period motifs at the beginning and ending parts of this work process since the sensor data from operations 3 to 10 (all screwing actions) were similar to each other. Recall that period motifs are defined as occurring only once per work period, making it unlikely to discover good candidates for them amongst so many operations that 


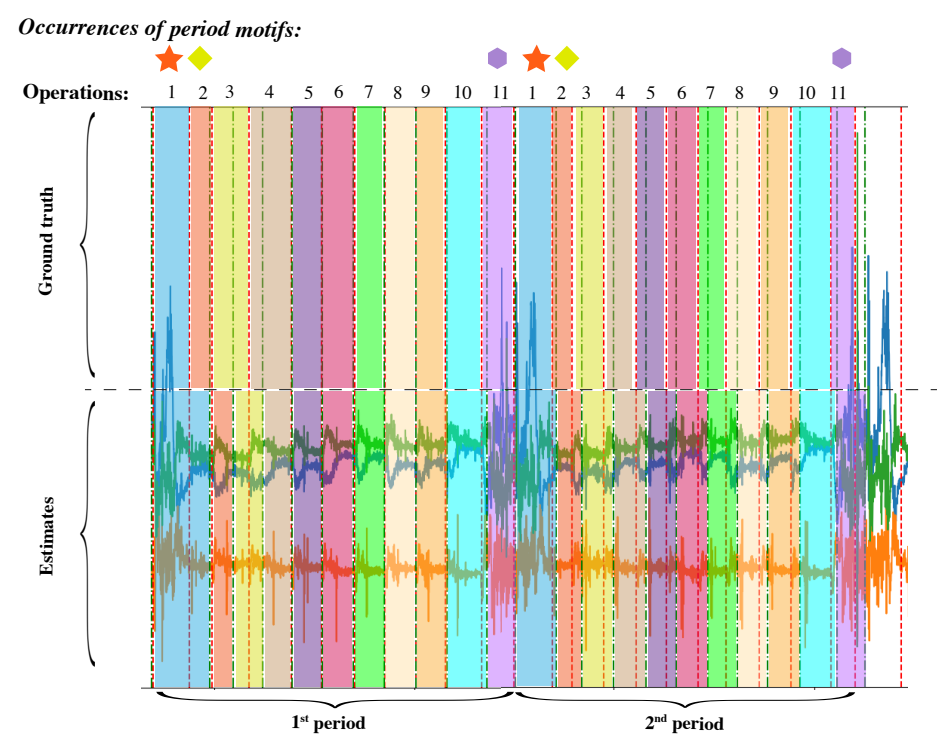

Fig. 17. Example output from W/o-Action for the first and second periods of work process $E$

Table 3. Computation time $[\mathrm{s}]$ of Proposed

\begin{tabular}{|c|c|c|c|c|c|c|c|c|c|}
\hline & & & & \multicolumn{4}{|c|}{ Tracking Operation Start Times } & \multirow[b]{2}{*}{ Others } & \multirow[b]{2}{*}{ Total } \\
\hline & $\begin{array}{c}\text { Preprocess } \\
\text { (HDP-HMM) }\end{array}$ & $\begin{array}{c}\text { Motif } \\
\text { Discovery }\end{array}$ & $\begin{array}{c}\text { Tracking Period } \\
\text { Motifs }\end{array}$ & $\begin{array}{l}\text { Trend-based } \\
\text { Score }\end{array}$ & $\begin{array}{l}\text { Period-motif } \\
\text { Score }\end{array}$ & $\begin{array}{l}\text { Action-motif } \\
\text { Score }\end{array}$ & $\begin{array}{c}\text { Manipulate } \\
\text { Tree+Others }\end{array}$ & & \\
\hline work A & 353.5 & 3.9 & 6.2 & 0.007 & 1.6 & 3.5 & 39.3 & 4.1 & 412.1 \\
\hline B & 187.8 & 0.5 & 5.3 & 0.005 & 0.7 & 2.1 & 74.1 & 3.1 & 273.6 \\
\hline C & 230.2 & 0.5 & 6.9 & 0.009 & 1.8 & 3.5 & 181.5 & 3.0 & 427.3 \\
\hline D & 189.8 & 0.5 & 3.0 & 0.002 & 1.3 & 3.2 & 69.7 & 3.0 & 270.5 \\
\hline $\mathbf{E}$ & 224.5 & 0.5 & 4.0 & 0.014 & 2.2 & 4.3 & 46.8 & 3.5 & 285.7 \\
\hline $\bar{F}$ & 210.8 & 0.5 & 0.9 & 0.013 & 1.7 & 3.8 & 56.5 & 4.3 & 278.5 \\
\hline
\end{tabular}

Table 4. Computation time $[\mathrm{s}]$ of Xia2019

\begin{tabular}{|l||l|l|l|l|l|}
\hline & $\begin{array}{l}\text { Preprocess } \\
\text { (HDP-HMM) }\end{array}$ & Motif Discovery & $\begin{array}{l}\text { Tracking Operation } \\
\text { Start Times }\end{array}$ & Others & Total \\
\hline \hline work A & 362.8 & 0.7 & 1914.4 & 9.4 & $\mathbf{2 2 8 7 . 3}$ \\
\hline B & 223.0 & 0.1 & 3038.0 & 1.0 & $\mathbf{3 2 6 2 . 1}$ \\
\hline C & 221.3 & 0.1 & 1450.0 & 4.0 & $\mathbf{1 6 7 5 . 4}$ \\
\hline D & 194.7 & 0.1 & 4558.8 & 2.7 & $\mathbf{4 7 5 6 . 3}$ \\
\hline E & 188.2 & 0.1 & 753.2 & 3.1 & $\mathbf{9 4 4 . 8}$ \\
\hline F & 194.6 & 0.1 & 892.7 & 27.0 & $\mathbf{1 1 1 4 . 4}$ \\
\hline
\end{tabular}

consist largely of similar actions. Therefore, it was difficult to precisely detect these operations without using the action-motif score. As above, because action motifs are effective only in work processes with few useful period motifs, we could not find the significant difference between Proposed and W/o-Action.

4.3.7 Computation Time. Tables 3 and 4 show computation times for each process for Proposed and Xia2019 along with their total computation times. As shown in these results, the total computation time for Proposed 
is much shorter than that of Xia2019, which relies on a computationally expensive technique, i.e., DTW ${ }^{2}$. In contrast, Proposed leverages period and action motifs to quickly detect the start times for the operations. In fact, the total computation time for Proposed is shorter than the duration of the input sensor data, indicating the possibility for its use in real-time unsupervised activity recognition. Since the computation time for HDPHMM currently dominates the overall computation time for Proposed, we plan to replace HDP-HMM with a lightweight technique as a part of our future work.

\section{CONCLUSION}

In this study, we have proposed a new unsupervised factory activity recognition method that is robust to outlier sensor data segments. Our method robustly estimates the start time for each operation in a work period by leveraging two types of motifs: period motifs and action motifs. We evaluated the proposed method using sensor data collected from workers in actual factories and achieved state-of-the-art performance. In addition, we confirmed that the computation time of the proposed method is significantly shorter than that of the prior state-of-the-art method. As a part of our future work, we plan to implement a real-time factory activity recognition system based on our method in an actual factory.

\section{REFERENCES}

[1] Mario Aehnelt and Sebastian Bader. 2015. Information assistance for smart assembly stations. In the 7th International Conference on Agents and Artificial Intelligence (ICAART 2015), Vol. 2. 143-150.

[2] Mario Aehnelt, Enrico Gutzeit, and Bodo Urban. 2014. Using activity recognition for the tracking of assembly processes: Challenges and requirements. In WOAR 2014. 12-21.

[3] Mario Aehnelt and Bodo Urban. 2015. The knowledge gap: providing situation-aware information assistance on the shop floor. In $H C I$ in Business. 232-243.

[4] Mario Aehnelt and Karoline Wegner. 2015. Learn but work!: towards self-directed learning at mobile assembly workplaces. In the 15th International Conference on Knowledge Technologies and Data-driven Business. 17.

[5] Sebastian Bader, Frank Krüger, and Thomas Kirste. 2015. Computational causal behaviour models for assisted manufacturing. In the 2nd international Workshop on Sensor-based Activity Recognition and Interaction. 14.

[6] Martin Bauer, Lamine Jendoubi, and Oliver Siemoneit. 2004. Smart Factory-Mobile Computing in Production Environments. In the MobiSys 2004 Workshop on Applications of Mobile Embedded Systems (WAMES 2004).

[7] Eugen Berlin and Kristof Van Laerhoven. 2012. Detecting leisure activities with dense motif discovery. In Proceedings of the 2012 ACM Conference on Ubiquitous Computing. 250-259.

[8] Mark Blum, Alex Sandy Pentland, and Gehrard Tröster. 2006. Insense: Interest-based life logging. IEEE Multimedia 13, 4 (2006), $40-48$.

[9] Tâm Huynh, Mario Fritz, and Bernt Schiele. 2008. Discovery of activity patterns using topic models. In UbiComp 2008. 10-19.

[10] Matthew J. Johnson and Alan S. Willsky. 2013. Bayesian Nonparametric Hidden Semi-Markov Models. Journal of Machine Learning Research 14, 1 (2013), 673-701.

[11] Eamonn Keogh, Kaushik Chakrabarti, Michael Pazzani, and Sharad Mehrotra. 2001. Dimensionality reduction for fast similarity search in large time series databases. Knowledge and Information Systems 3, 3 (2001), 263-286.

[12] Quan Kong, Takuya Maekawa, Taiki Miyanishi, and Takayuki Suyama. 2016. Selecting home appliances with smart glass based on contextual information. In Proceedings of the 2016 ACM International foint Conference on Pervasive and Ubiquitous Computing (UbiComp 2016). ACM, 97-108.

[13] Oliver Korn, Albrecht Schmidt, and Thomas Hörz. 2013. The potentials of in-situ-projection for augmented workplaces in production: a study with impaired persons. In CHI'13 Extended Abstracts. 979-984.

[14] Joseph Korpela, Kazuyuki Takase, Takahiro Hirashima, Takuya Maekawa, Julien Eberle, Dipanjan Chakraborty, and Karl Aberer. 2015. An energy-aware method for the joint recognition of activities and gestures using wearable sensors. In International Symposium on Wearable Computers (ISWC 2015). 101-108.

[15] Heli Koskimäki, Ville Huikari, Pekka Siirtola, Perttu Laurinen, and Juha Röning. 2009. Activity recognition using a wrist-worn inertial measurement unit: A case study for industrial assembly lines. In 17th Mediterranean Conference on Control and Automation (MED 2009). $401-405$.

\footnotetext{
${ }^{2}$ This method employs DTW to compute the consistency of sensor data for an estimated operation between different work periods.
} 
[16] Frank Krüger, Martin Nyolt, Kristina Yordanova, Albert Hein, and Thomas Kirste. 2014. Computational state space models for activity and intention recognition. A feasibility study. PloS one 9, 11 (2014).

[17] Jonathan Lester, Tanzeem Choudhury, and Gaetano Borriello. 2006. A practical approach to recognizing physical activities. In Pervasive 2006. 1-16.

[18] Jessica Lin, Eamonn Keogh, Stefano Lonardi, and Pranav Patel. 2002. Finding motifs in time series. In The 2nd Workshop on Temporal Data Mining. 53-68.

[19] Bruno Lotter and Hans-Peter Wiendahl. 2013. Montage in der industriellen Produktion: Ein Handbuch für die Praxis. Springer-Verlag.

[20] Dominik Lucke, Carmen Constantinescu, and Engelbert Westkämper. 2008. Smart factory-a step towards the next generation of manufacturing. In Manufacturing systems and technologies for the new frontier. Springer, 115-118.

[21] Paul Lukowicz, Holger Junker, Mathias Stäger, Thomas von Bueren, and Gerhard Tröster. 2002. WearNET: A distributed multi-sensor system for context aware wearables. In Ubicomp 2002. 361-370.

[22] Paul Lukowicz, Jamie A Ward, Holger Junker, Mathias Stäger, Gerhard Tröster, Amin Atrash, and Thad Starner. 2004. Recognizing workshop activity using body worn microphones and accelerometers. In Pervasive 2004. 18-32.

[23] Takuya Maekawa, Yasue Kishino, Yutaka Yanagisawa, and Yasushi Sakurai. 2012. Recognizing handheld electrical device usage with hand-worn coil of wire. In Pervasive 2012. 234-252.

[24] Takuya Maekawa, Yasue Kishino, Yutaka Yanagisawa, and Yasushi Sakurai. 2012. WristSense: wrist-worn sensor device with camera for daily activity recognition. In 2012 IEEE International Conference on Pervasive Computing and Communications Workshops (PERCOM Workshops). 510-512.

[25] Takuya Maekawa, Daisuke Nakai, Kazuya Ohara, and Yasuo Namioka. 2016. Toward practical factory activity recognition: unsupervised understanding of repetitive assembly work in a factory. In UbiComp 2016. 1088-1099.

[26] Takuya Maekawa and Shinji Watanabe. 2011. Unsupervised activity recognition with user's physical characteristics data. In International Symposium on Wearable Computers (ISWC 2011). 89-96.

[27] David Minnen, Thad Starner, Irfan Essa, and Charles Isbell. 2006. Discovering characteristic actions from on-body sensor data. In 2006 10th IEEE international symposium on wearable computers. IEEE, 11-18.

[28] Francisco Javier Ordóñez Morales and Daniel Roggen. 2016. Deep convolutional feature transfer across mobile activity recognition domains, sensor modalities and locations. In The 2016 ACM International Symposium on Wearable Computers (ISWC). ACM, 92-99.

[29] Fernando Moya Rueda, René Grzeszick, Gernot A Fink, Sascha Feldhorst, and Michael Ten Hompel. 2018. Convolutional neural networks for human activity recognition using body-worn sensors. In Informatics, Vol. 5. Multidisciplinary Digital Publishing Institute, 26.

[30] Agnieszka Radziwon, Arne Bilberg, Marcel Bogers, and Erik Skov Madsen. 2014. The Smart Factory: Exploring adaptive and flexible manufacturing solutions. Procedia Engineering 69 (2014), 1184-1190.

[31] Juhi Ranjan and Kamin Whitehouse. 2015. Object hallmarks: Identifying object users using wearable wrist sensors. In UbiComp 2015. 51-61.

[32] Hiroaki Sakoe and Seibi Chiba. 1978. Dynamic programming algorithm optimization for spoken word recognition. IEEE Transactions on Acoustics, Speech and Signal Processing 26, 1 (1978), 43-49.

[33] Andrea Rosales Sanabria and Juan Ye. 2020. Unsupervised domain adaptation for activity recognition across heterogeneous datasets. Pervasive and Mobile Computing (2020), 101147.

[34] Thomas Stiefmeier, Georg Ogris, Holger Junker, Paul Lukowicz, and Gerhard Tröster. 2006. Combining motion sensors and ultrasonic hands tracking for continuous activity recognition in a maintenance scenario. In 10th IEEE International Symposium on Wearable Computers (ISWC 2006). 97-104.

[35] Thomas Stiefmeier, Daniel Roggen, and Gerhard Tröster. 2007. Fusion of string-matched templates for continuous activity recognition. In 11th IEEE International Symposium on Wearable Computers (ISWC 2007). 41-44.

[36] Edison Thomaz, Irfan Essa, and Gregory D Abowd. 2015. A practical approach for recognizing eating moments with wrist-mounted inertial sensing. In UbiComp 2015. 1029-1040.

[37] Jamie A Ward, Paul Lukowicz, and Gerhard Tröster. 2005. Gesture spotting using wrist worn microphone and 3-axis accelerometer. In The 2005 foint Conference on Smart Objects and Ambient Intelligence: Innovative context-aware services: usages and technologies. 99-104.

[38] Qingxin Xia, Atsushi Wada, Joseph Korpela, Takuya Maekawa, and Yasuo Namioka. 2019. Unsupervised Factory Activity Recognition with Wearable Sensors Using Process Instruction Information. Proceedings of the ACM on Interactive, Mobile, Wearable and Ubiquitous Technologies 3, 2 (2019), 60.

[39] Juan Ye, Simon Dobson, and Franco Zambonelli. 2020. XLearn: Learning Activity Labels across Heterogeneous Datasets. ACM Transactions on Intelligent Systems and Technology (TIST) 11, 2 (2020), 1-28.

[40] Kristina Yordanova. 2018. Extracting planning operators from instructional texts for behaviour interpretation. In foint German/Austrian Conference on Artificial Intelligence (Künstliche Intelligenz). Springer, 215-228. 
(a)

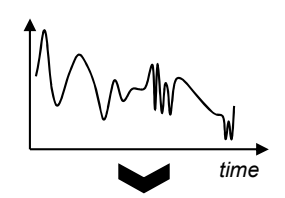

(b)

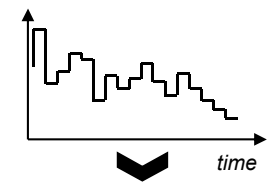

(c)

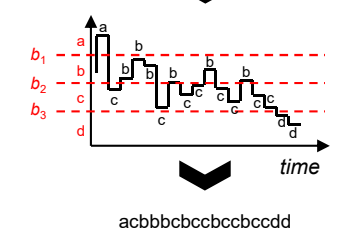

Fig. 18. Symbolizing sensor data. (a) Example original sensor data, (b) sensor data after performing PAA, and (c) sensor data after symbolization according to [18]. (a)

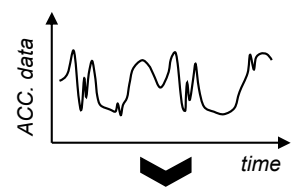

(b)

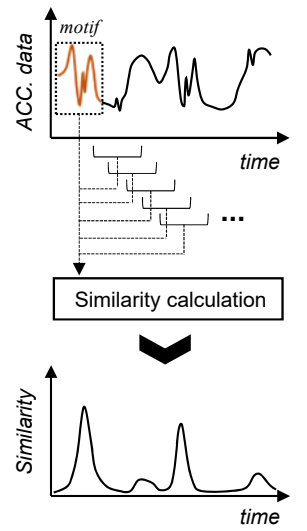

Fig. 19. Calculating the similarity between segments of time series data. (a) Example original sensor data, (b) calculating the similarity between a motif and consecutive data segments using a sliding window, and (c) resulting output time series of similarity values.

\section{APPENDIX}

\subsection{Preprocessing Sensor Data}

We first standardize and then discretize (symbolize) the input acceleration data using the methods described in [18]. We start by standardizing the raw time series data and then downsample the data using piecewise aggregate approximation (PAA) [11], where the time series is divided into equally-sized frames with each frame then represented by a single value computed as the mean value of that frame, as shown in Figure 18 (b). We then convert the downsampled time series data into a series of symbols according to the method in [18], which divides the full range of values for the time series data into multiple bins, with each value in the time series assigned a symbol based on the bin that contains its value. For example, in Figure 18 (c), the $c$ bin corresponds to values between $b_{2}$ and $b_{3}$, so any value in that range would be replaced by the symbol $c$.

We next identify likely starting locations for operations by segmenting the sensor data based on trend changes in the data using a Bayesian nonparametric version of HMM called the hierarchical Dirichlet process HMM (HDP-HMM) [10]. HDP-HMM is a non-parametric method for generating HMMs that can predict a posterior distribution for the number of states in an HMM, enabling it to segment a time series using an HMM without knowledge about the number of states in that HMM. Refer to [10] for more details about HDP-HMM.

\subsection{Scoring Functions for Selecting Period Motifs}

Here we explain the scoring functions used for selecting period motifs in detail. 
(1) We compute the first score for a candidate period motif at time $t$ as follows:

$$
s_{p k}=\max \left(\operatorname{peak}\left(\operatorname{seg}_{1}\right)\right)-\operatorname{mean}\left(\operatorname{peak}\left(\operatorname{seg}_{2}\right)\right),
$$

where $\operatorname{seg}_{2}$ is a data segment over $(t, t+0.8 \cdot \mathcal{M}$.min_lead_time $]$ within $\mathrm{S}, \operatorname{seg}_{1}$ is a segment over $[t+0.8$. M.min_lead_time, $\left.t_{\text {init }}\right]$ within S, M.min_lead_time is the shortest standard lead time described in the work model $\mathcal{M}$, and peak (seg) detects peaks (local maxima) within seg by simply computing the derivative. Here, $s e g_{1}$ shows a segment that can contain occurrences of the motif and is used to obtain similarity values at occurrences of the motif. In addition, $\operatorname{seg}_{2}$ shows a segment that does not contain occurrences of the motif and is used to obtain similarity values other than the occurrences of the motif. With this score, we can capture the difference between the similarity value at the highest peak and those at the other time slices.

We also store a parameter $t h_{p m}=0.8 \cdot \max \left(\operatorname{peak}\left(\operatorname{seg}_{1}\right)\right)$ for each candidate motif, which gives us a threshold to use when determining if similarity values for sensor data should be considered to be an occurrence of the motif during the procedure used when tracking period motifs.

(2) We compute the similarity value of the candidate motif as the second score.

$$
s_{u q}=\max \left(\operatorname{peak}\left(\operatorname{seg}_{1}\right)\right) \text {. }
$$

\subsection{Scoring Function for Selecting Action Motifs}

Here we explain the scoring function used for selecting action motifs in detail.

We calculate the score based on the average prominence of peaks in a segment of similarity time series that is expected to contain the initial work period:

$$
s_{p r}=m e a n\left(\operatorname{prominence}\left(\operatorname{peak}\left(\operatorname{seg}_{3}\right)\right)\right),
$$

where $\operatorname{seg}_{3}$ shows a segment that contains the initial work period.

\subsection{Determining $t_{f}$ when generating $\mathcal{G}$}

$t_{f}$ is the time when $\mathrm{S}$ first exceeds $t h_{p m}$ after $t\left(p_{i}, n\right)+d_{f} \cdot$ M.min_lead_time plus a margin. Note that, when an outlier segment occurs after $t\left(p_{i}, n\right)$ and a sub-segment within the outlier segment exhibits a high similarity value with the motif of interest, the value of $t_{f}$ may be erroneously set as a time before the next occurrence of the motif. To help cope with this, the particle filters conditionally apply a correction to $t_{f}$ based on the results for $t_{f}$ from the other particle filters. Briefly speaking, when $t_{f}-t\left(p_{i}, n\right)$ (i.e., temporal distance between $t_{f}$ and the timestamp of $p_{i}$ ) for some particle filter is much smaller than $t_{f}-t\left(p_{i}, n\right)$ for other particle filters, we increase $t_{f}$ for this particle filter based on $t_{f}-t\left(p_{i}, n\right)$ from the base particle filter.

\subsection{Score Calculation for Tracking Period Motifs}

Similarity score: A sensor data segment corresponding to $p_{j}$ should be similar to the segment corresponding to the first occurrence of the motif. Therefore, we simply use the similarity value at $t\left(p_{j}, n+1\right)$ as follows.

$$
s_{\text {sim }}\left(p_{j}\right)=\mathrm{S}_{t\left(p_{j}, n+1\right)}
$$

Consistency score: We calculate this score by referring to the base particle filter and the temporal structure model $\mathcal{T}$. Because the temporal relationship between the occurrences of period motifs should be consistent throughout work periods, we calculate this score using the following equation:

$$
s_{c o n}\left(p_{j}\right)=\frac{1}{\mid \mathcal{T}\left(p f_{b} . \text { motif }, p f . \text { motif }\right)-\left(o\left(p f_{b} . \text { motif }, n+1\right)-t\left(p_{j}, n+1\right)\right) \mid},
$$


where $p f_{b}$ is the base particle filter, $p f$.motif is a motif that a particle filter $p f$ tracks, and $o\left(p f_{b}\right.$.motif, $\left.n+1\right)$ is the posterior estimate of the $n+1$-th occurrence of the base motif (refer to Section 3.5.4). Therefore, the above function calculates the consistency in the temporal distance between the occurrences of the motifs $\left(o\left(p f_{b}\right.\right.$.motif,$\left.\left.n+1\right)-t\left(p_{j}, n+1\right)\right)$ by referring to the history of the time difference in the temporal structure model $\left(\mathcal{T}\left(p f_{b} \text {.motif, pf.motif }\right)\right)^{3}$.

\subsection{Score Calculation for Tracking Operation Start Times}

Trend-based score: This score enables us to detect operation start times based on the trend changes discovered by HDP-HMM. This score is computed based on the temporal distance between the timestamp of a particle and the timestamp of the closest trend change in $\mathrm{C}$ as follows:

$$
\left.\operatorname{CumulativeDist}\left(p_{j}, n, k, \mathbf{C}\right)=\sum_{m=2}^{n+1} \operatorname{Dist}\left(t\left(p_{j}, m, k\right), \mathbf{C}\right)\right),
$$

where $t\left(p_{j}, m, k\right)$ is the timestamp for the ancestor particle of $p_{j}$ corresponding to the $m$-th operation in the $k$-th work period (when $m$ is equal to $n+1$, it is the timestamp for $p_{j}$ itself) and

$$
\operatorname{DisT}(t, \mathbf{C})=\min _{c \in \mathbf{C}}|t-c| \text {. }
$$

The inverse of this distance is then used as the score.

Period-motif score: The particle filters for period motifs output posterior estimates for the occurrences of the period motifs. This score evaluates the quality of a series of estimated operation start times based on the consistency of period-motif locations in the estimated work periods, based on our expectation that the temporal distances between operation start times and occurrences of period motifs should be consistent. We start by computing two values for the start time of the particle of interest (i.e., $\left.t\left(p_{j}, n, k\right)\right)$ that measure its distances from the occurrence of the motif just before $t\left(p_{j}, n, k\right)$ and the occurrence of the motif just after $t\left(p_{j}, n, k\right)$. We then use these distances to compare the $k$-th and $k-1$-th occurrences of a motif by computing the squared difference between each value for $k$-th and $k$-1-th occurrences. The inverse of the sum of the squared differences is then used as the score.

Action-motif score: A similarity time series is given for each action motif, which represents the occurrences of that action motif. This score then evaluates the quality of an estimated series of operation start times based on the consistency of the waveforms for the action motifs' similarity time series between $t\left(p_{i}, n, k\right)$ and $t\left(p_{j}, n+1, k\right)$, i.e., a segment corresponding to the $n$-th operation, because waveforms for corresponding segments in different work periods should be similar to each other when the start and end times of the operations are properly tracked. We extract statistical features from the segments for $k$ and $k-1$ to form two feature vectors and then compute the Euclidean distance between the two vectors. The following statistical features are extracted from each segment: (i) standard deviation, (ii) skewness, (iii) kurtosis, (iv) \#peaks divided by the duration of the segment, and (v) mean. The inverse of the distance is then used as the score.

\subsection{Evaluation Using Data from the Logistics Domain}

The proposed method is designed to recognize structured repetitive work. Our main experiment investigated the effectiveness of our method using factory activity data collected in actual factories. Here we test our method on sensor data collected from the logistics domain. This acceleration dataset was collected from a worker in a delivery center using a smartwatch. The worker performed packing tasks repetitively, and the work mainly

\footnotetext{
${ }^{3}$ To deal with an optional operation, we have $\mathcal{T}\left(m_{i}, m_{j}\right)$ for each possible pattern of the process (i.e., the number of patterns is identical to the number of leaf nodes in the operation flow model).
} 


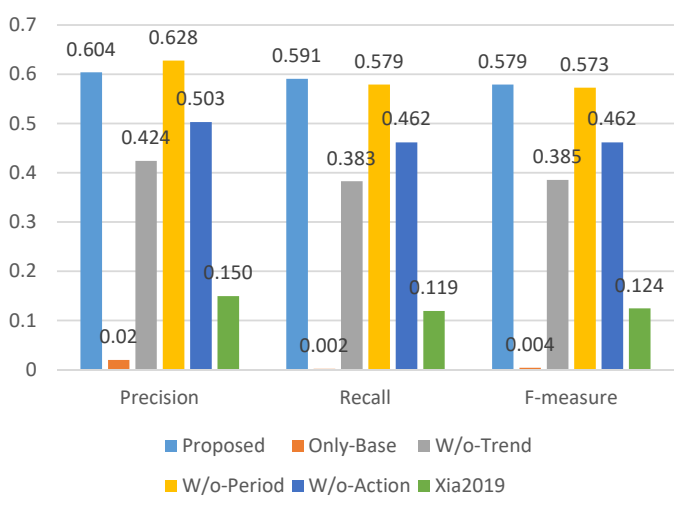

Fig. 20. Precision, recall, and F-measure for each method when using data from the logistics domain.

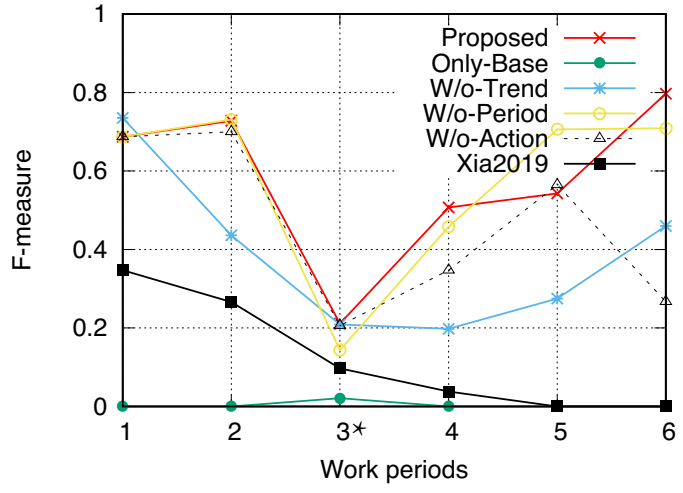

Fig. 21. Transitions in F-measures for each method when using data from the logistics domain. The $\left({ }^{*}\right)$ in the horizontal axis indicates periods with outliers.

consisted of seven operations: pick up items, replace labels, assemble a box, pack items, close the box, scan the label, and attach an address label. The approximate duration of each work period is 45 seconds. Note that packing work from the logistics domain is different from the type of work assumed in this study, because their operations differ depending on items to be packed. For example, the duration of the "pack items" operation changes depending on the number of items packed in the same box. In addition, the size of a box depends on the size of an item to be packed, resulting in differences in the sensor data for the same operation in different work periods. Note that, because there are some unrelated events between two consecutive periods, we generated this data set by removing segments corresponding to these events.

Figure 20 shows the precision, recall, and F-measure for each method. Figure 21 breaks down the average F-measures from Figure 20 into the individual F-measures for each work period. As shown in these results, the overall accuracies of the methods are poorer than those in the factory work domain. This is because the durations of operations depend greatly on the items to pack as described above. However, Proposed achieved the highest F-measure, with W/o Period performing similarly. The accuracy of W/o Period was similar to that of Proposed, because we could not find period motifs around the middle portion of each period, where the sensor data of corresponding operations differed in each period. Figure 22 shows an example of the output from Proposed. As shown in the figure, Proposed could successfully track period motifs, and achieved high accuracy in the second period, with the exception of the second operation (replace labels). During the second operation, the worker issues labels using a machine. Because the time needed to issue the labels depends greatly on the number of items, the duration of this operation varies greatly. Proposed also failed to detect an outlier between the fourth and fifth operations in the third period, because it failed to detect the second and third operations and period motifs were found only around the initial and final portions of each period. Similar to the second operation, the duration of the third operation (assemble a box) varies greatly, because it is affected by the size and complexity of the box. 
- Xia et al.

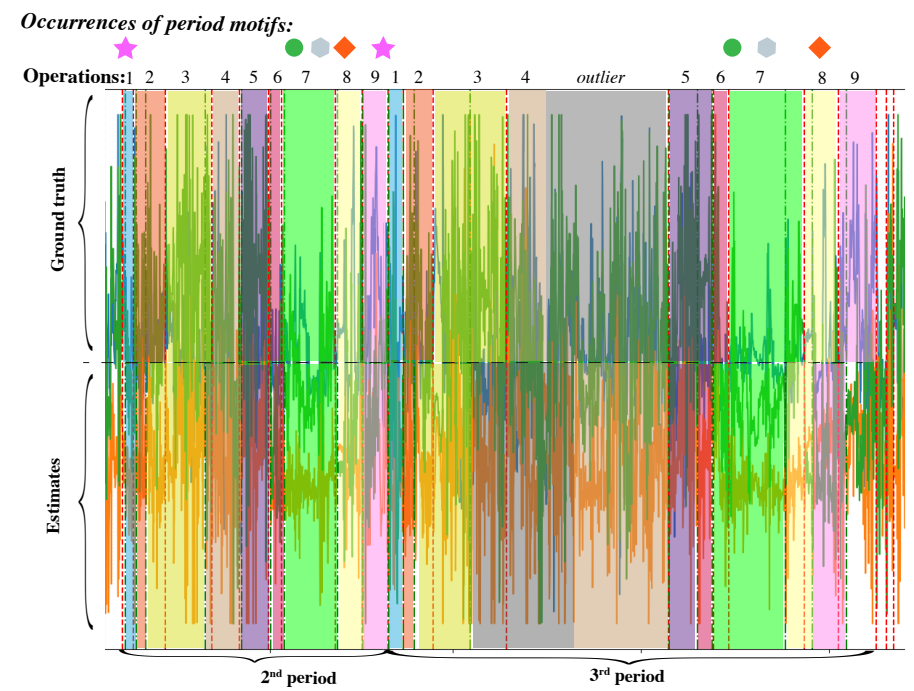

Fig. 22. Example output from Proposed for the second and third periods of data from the logistics domain. 